\title{
Breast Cancer: Modelling and Detection
}

\author{
D.J. GAVAGHAN ${ }^{\mathrm{a}}$, J.M. BRADY ${ }^{\mathrm{b}}$, C.P. BEHRENBRUCH ${ }^{\mathrm{b}}$, R.P. HIGHNAM ${ }^{\mathrm{c}}$ and P.K. MAINI ${ }^{\mathrm{d}, *}$ \\ ${ }^{\mathrm{a}}$ Computing Laboratory, Wolfson Building, Parks Road, Oxford OX1 3QD, UK; ${ }^{\mathrm{b}}$ Department of Engineering Science, Parks Road, Oxford OX1 3PJ, UK; \\ ${ }^{\mathrm{c}}$ OXIVA, Oxford Centre For Innovation, Mill Street, Oxford OX2 OJX, UK; ${ }^{\mathrm{d}}$ Centre for Mathematical Biology, Mathematical Institute, University of \\ Oxford, 24-29 St Giles', Oxford OX1 3LB, UK
}

(Received 7 October 2000; In final form 23 April 2001)

\begin{abstract}
This paper reviews a number of the mathematical models used in cancer modelling and then chooses a specific cancer, breast carcinoma, to illustrate how the modelling can be used in aiding detection. We then discuss mathematical models that underpin mammographic image analysis, which complements models of tumour growth and facilitates diagnosis and treatment of cancer. Mammographic images are notoriously difficult to interpret, and we give an overview of the primary image enhancement technologies that have been introduced, before focusing on a more detailed description of some of our own recent work on the use of physics-based modelling in mammography. This theoretical approach to image analysis yields a wealth of information that could be incorporated into the mathematical models, and we conclude by describing how current mathematical models might be enhanced by use of this information, and how these models in turn will help to meet some of the major challenges in cancer detection.
\end{abstract}

Keywords: Breast cancer; Tumour; Image processing; Mathematical models

\section{INTRODUCTION}

Cancer is the second biggest killer in the western world and its incidence is rapidly increasing. Despite the huge proliferation of experimental data and clinical treatments, there has been no decrease in the death rates caused by the most common cancers. This is despite the immense progress that has been made in understanding the causes and progression of cancer, particularly from the perspective of biology. There have been concomitant developments in drugs, chemotherapies and radiotherapies which together have meant that several kinds of cancer now have very positive prognoses.

However, much remains to be done on the modelling, early diagnosis (critical for improved prognosis) and effective treatment and treatment planning of cancer. Mathematics, in several different forms, is increasingly the basis of major developments across a range of disciplines in the understanding of cancer. The mathematics ranges from differential equations that model aspects of tumour growth to models of the formation of mammographic (X-ray) images, as well as newer imaging modalities such as contrast-enhanced MRI and ultrasound.

To date, the mathematics of tumour formation and spread has developed largely independently of the mathematics that underpins image analysis. This paper aims to illustrate how these two developments may usefully be integrated. We therefore begin with a review of the mathematics of tumour growth, before presenting a brief introduction to the mathematics of image analysis. This allows us to go on to discuss a number of situations in which the mathematical methods and the problems mentioned might be usefully combined.

The review of the mathematical literature given in "Previous mathematical models" describes previous models of cancer growth and proliferation. Over the past 30 years, a number of mathematical models have been proposed to attempt to describe various key stages of tumour development. However, there is still no basic consensus model of tumour growth and survival, metastasis (the process whereby potentially fatal secondary tumours are formed from a primary tumour), tumour angiogenesis (whereby nutrients are diverted to the tumour), or the breakdown of the extracellular matrix

*Corresponding author. 
tumour cells. One of the major challenges of the next decade is to develop biologically realistic mathematical models which clarify these fundamental processes and which can predict new strategies of clinical therapy.

Although some of this modelling work has contributed towards the search for non-invasive treatments of cancer, the most effective therapeutic approach remains the detection and excision of the malignant tumour and the surrounding tissue. In no area is this approach more emotionally traumatic for the patient than in breast cancer, and in this case, the early detection of cancerous growths is vital not only to improve the chance of survival but also to minimise the disfigurement caused by subsequent surgery. In "Breast cancer detection" the section, we sketch a number of recent advances in mammographic image analysis, before focusing on a more detailed description of some of our own recent work in mammography, which is based on modelling of the underlying physics of the image processing techniques, primarily for mammography.

In addition to providing a vitally important tool in identifying tumours, this theoretical approach can also be used to generate more realistic descriptions of both the composition and mechanics of breast or other types of tissue. In the "Enhancement of image processing through mathematical models" section we suggest ways in which this information might be incorporated into the existing mathematical models to give more realistic and useful descriptions of the physiology, and describe the advantages that would accrue in terms of the detection and treatment of cancer.

\section{PREVIOUS MATHEMATICAL MODELS}

In vivo cancer growth is a complicated phenomenon involving many inter-related processes. Solid tumour growth occurs in two distinct phases, initial growth being characterised as the avascular phase, later growth as the vascular phase. The transition from avascular growth to vascular growth depends upon the crucial process of neovascularisation and is necessary for the tumour to attain nutrients and dispose of waste products. To achieve vascularisation, tumour cells secrete a diffusible substance known as tumour angiogenesis factor (TAF) into the surrounding tissues. This has the effect of stimulating nearby capillary blood vessels to grow towards and penetrate the tumour, re-supplying the tumour with vital nutrient (Chaplain and Stuart, 1993). Invasion and metastasis can now take place. By the time a tumour has grown to a size whereby it can be detected by, in the case of breast-cancer, simple self-examination, there is a strong likelihood that it has already reached the vascular growth phase. The primary aim of screening and the associated image enhancement technologies is therefore to detect cancers prior to this stage.

The initial avascular growth phase can be studied in the laboratory by culturing cancer cells in the form of three-dimensional multicell spheroids (see, for example, Sutherland (1988)). It is well known that these spheroids, whether grown from established tumour cell lines or actual in vivo tumour specimens, possess growth kinetics which are very similar to in vivo tumours. Typically, these avascular nodules grow to a few millimetres in diameter. Cells towards the centre, being deprived of vital nutrients, die and give rise to a necrotic core. Proliferating cells can be found in the outer three to five cell layers. Lying between these two regions is a layer of quiescent cells, a proportion of which can be recruited into the outer layer of proliferating cells.

Many models have been proposed to account for the early stages of avascular growth. Early models considered simple diffusion of nutrients and growth inhibitory factors (Greenspan, 1972, 1974; Adam, 1987) and showed that a number of different hypotheses on chemical kinetics and mode of diffusion could result in concentration profiles that were not incompatible with experimental data (Chaplain et al., 1994). A comprehensive account of modelling avascular tumour growth is given in (Marušić et al., 1994), where several mathematical models for growing multicellular spheriods are compared with experimental data. Byrne and Chaplain (1996a) developed a mathematical model for the growth of a multicellular spheriod that comprised a central core of necrotic cells surrounded by an outer annulus of proliferating cells. Their model distinguished between necrosis and apoptosis and considered the effect of nutrients and growth inhibitors.

More detailed and sophisticated models were later introduced. For example, Byrne (1997) assumed that growth rate depends on the external supply of nutrients and the intercellular adhesion forces which maintain tumour compactness. Her model equations consider the spatiotemporal dynamics of nutrient concentration and pressure within the tumour. Ward and King (1997) describe a continuum of cells which, through local volume changes, create movement described by a velocity field. This approach results in the evolution of a three layer tumour structure of proliferating, quiescent and necrotic cells. Stott et al. (1999) simulated growth of an avascular tumour embedded in normal tissue by evoking energy minimization to effect cell sorting between normal and tumour cell types, also leading to the formation of a threelayered tumour structure. In Byrne and Chaplain (1996a) it was shown how a radially symmetric avascular tumour could become unstable, possibly leading to tumour invasion.

When a tumour reaches a critical size, it begins to release so called angiogenesis factors into surrounding tissue which diffuse towards the neighbouring vasculature creating a chemical gradient. Capillary sprouts are formed as the blood vessel wall breaks down and releases endothelial cells. The sprouts then grow towards the tumour and each other, and loops are formed in a process known as anastomosis, establishing a blood supply for the tumour. 
The process of angiogenesis has been extensively modelled by Chaplain and co-workers, who consider both continuous and discrete mathematical models which take into account the complex interaction of chemotactic agents, and endothelial cell-extracellular matrix interactions via the adhesive molecule fibronectin. By appropriately discretizing their continuum partial differential equation model, they develop a biased random-walk model, which allows them to track the motion of individual cells. This provides a model framework which can incorporate anastomosis, mitosis and branching. The two- and three-dimensional capillary networks generated by these models compare very favourably with those observed in in vivo experiments (Anderson and Chaplain, 1998). This latter approach can also be used to address metastasis and it has been shown that whereas a continuum model will predict a spreading mass, the probabilistic-type model does show single cells breaking away from the central mass, reminiscent of observations on mammograms showing breast cancer (Anderson et al., 2000).

The above models mostly consider the medium in which tumours grow to be homogeneous. However, in vivo, tissues have a high degree of fine-scaled spatial structure and the paper by Anderson et al. (2000) investigates the effects of spatial heterogeneity. Mathematical models of cancer chemotherapy have also been developed to investigate, for example, the use of targeted antibody-enzyme conjugates for the selective activation of anti-cancer prodrugs (Jackson et al., 2000) and the effects of drug resistance on the optimal scheduling of drugs (Murray, 1997).

Swanson (1999) and Swanson et al. (2000) considered tissue heterogeneity in the case of brain gliomas, which are generally highly diffuse. These are usually detected using computerised tomography (CT) and magnetic resonance imaging (MRI). However, even after surgical excision well beyond the visible tumour boundary, a recurrence near the edge of resection ultimately results. This is because the presently available imaging techniques only detect a small proportion of the actual, highly diffuse, tumour.

Experiments in rats show that malignant glioma cells implanted in rat brain disperse more quickly along white matter tracts than grey matter. Swanson (1999) and Swanson et al. (2000) considered a reaction-diffusion model for glioma cell invasion on a two-dimensional anatomically accurate slice of brain tissue in which they imposed a spatially dependent cell diffusion coefficient to account for different cell motility rates in grey and white matter. Using numerical simulation, they characterised how the proportion of tumour detected depended on the cell diffusion coefficient and cell proliferation rate. They also showed that the heterogeneity within the brain caused the dynamics of tumour invasion to vary significantly depending on the initial location of the tumour. These results have important implications on how much tissue a surgeon should aim to remove when a tumour is detected.
The aforementioned models have resulted in a novel perspective on the different stages of tumour growth. Mathematically, the models all essentially amount to systems of differential equations. It is known that the spatiotemporal dynamics of such types of models can be dramatically changed if the environment is allowed to be spatially heterogeneous. For example, in Maini et al. (1992) the authors considered the problem of diffusiondriven instability in a system of reaction-diffusion equations. They found that if they chose a bifurcation parameter (in their case, the ratio of chemical diffusivities) to vary across a one-dimensional domain, the patterns exhibited by the system varied in amplitude and/or wavelength across the domain. In particular, they found that if the parameter was chosen to be above its bifurcation value only in a sub-interval of the domain, then it was possible for patterns to propagate into the domain where linear analysis would predict stability of the uniform steady state - a situation they described as "environmental instability" (Benson et al., 1993). A detailed weakly nonlinear bifurcation analysis for the two-dimensional case showed how degenerate bifurcation points could be split using a spatially varying parameter (Benson et al., 1998). These results show that environmental inhomogeneity could be an important regulator of biological pattern formation.

Understanding how the predictions of the above models depend on the (spatiotemporally) varying material properties of the matrix surrounding the growing tumour is one of the next key steps in the modelling effort.

\section{BREAST CANCER DETECTION}

The work of Swanson and co-workers illustrates the importance of tissue heterogeneity in mathematical models of cancer, and suggests that by using such models we might improve the efficacy of non-invasive cancer detection techniques which are based on image analysis. One of the most common cancers is breast cancer, which is developed by approximately $10 \%$ of women in first world countries during the course of their lives. Early detection of breast cancer gives the best chance of survival, and so breast self-examination is encouraged throughout the (Western) world. However, it has been shown that most women can only reliably detect a lump when it has reached a size-typically $0.75 \mathrm{~cm}$-at which it is likely already to have metastasised. While breast self-examination continues to be encouraged, not least because it raises the awareness of women to breast cancer, additional means of early detection are necessary.

To this end, screening programmes based on (X-ray) mammograms have been established in many countries. In the UK, for example, the current breast screening programme invites women between the ages of 50 and 70 for breast screening. The reason for restricting screening to women in this age group is that younger, pre-menopausal women have dense breasts whose milk-bearing tissues 
strongly attenuate X-rays, so that the resulting mammogram is often entirely white, and it is extremely difficult or impossible to detect small lesions. During the menopause, milk bearing tissue involutes into fat, which is radiotransparent, enabling high contrast images to be taken. The effectiveness of mammography for younger women has not yet been established. If a mammogram displays any suspicious signs, the woman is invited back to an assessment clinic where other views and imaging modalities are utilised. In other countries, for example the USA, France, and Germany, there is not (yet) a national screening programme; however, women are encouraged to have a mammogram regularly. The increased awareness of breast cancer has, contrary to almost all other forms of cancer, led to a slight increase in the detected incidence over the past 20 years.

It is important, at the outset of any discussion of mammographic image analysis, to emphasise the need for mathematical modelling, and the severity of the challenge.

- In order to reduce the radiation dose, the breasts are tightly compressed while a mammogram is taken. The compression depends qualitatively upon the operator's experience and judgement of the density of the woman's breasts. The compression typically varies by as much as $0.5 \mathrm{~cm}$ at successive mammograms. To date, there is no realistic model of breast compression. While such a model would have immediate application in image analysis, the complexity of breast anatomy means that models will inevitably be coarse approximations for the foreseeable future.

- Screening practice in the UK increasingly recommends that two different mammogram views are taken of each breast, namely cranio-caudal (CC) - head to toe-and mediolateral oblique (MLO) — shoulder to the opposite hip. The MLO view enables axillar involvement, a strong indicator of metastasis, to be detected in many cases. It has been demonstrated clinically that twoview mammography has significantly better diagnostic outcomes than single view mammography (as is practised in the screening programmes in several other countries). However, breasts are not cylindrical in cross-section, and the separation between the compression plates typically differs by $1 \mathrm{~cm}$ from $\mathrm{CC}$ and MLO. The difference in compression and the lack of a reasonable model of compression implies that it is difficult to combine the CC and MLO views into a three-dimensional representation. We have developed an initial computational model to combine these images, and it is sketched in "Matching between views" below.

- It was noted about 20 years ago that small deposits of calcium salts, called microcalcifications, are often the earliest indicators of the presence of a tumour. The detection of microcalcifications has been a major goal of screening programmes, hence of mammographic image analysis. Microcalcifications attenuate X-rays strongly (about 50 times more than normal tissue, cancerous tissue, or fat) and, though they are sometimes obscured by normal parenchymal tissue, they can often be detected as small regions of high contrast in mammograms.

\section{Mammographic Image Processing}

Mammograms are extremely complex images for a radiologist (or computer program) to assess. Partly, this reflects the intrinsic complexity of breast tissue. It also reflects the relatively poor signal-to-noise ratio of the images, the complexity of the image formation process, and the subtlety of the clinically significant signs that appear. While increasing numbers of women are demanding mammograms, there is increasing concern about the level of diagnostic accuracy of the radiologists who assess those mammograms. More importantly, there is growing concern over the low number of radiologists specialising in mammography. For these reasons, coupled with the tumbling costs of computing, improvements in software systems, and the increasing bandwidth of communications there is substantial and growing interest, both within the academic community and in industry, in developing mammographic image-analysis technologies to support a radiologist, either by prompting locations in an image, or acting as a "second reader". Indeed, evidence has recently been presented that shows that the combination of a radiologist and a Computer-Aided Detection (CAD) algorithm has significantly higher specificity and sensitivity than either working alone.

Initially, mammographic image analysis consisted simply of the application of standard image analysis techniques to digitised mammogram films. Such techniques comprise image enhancement, region segmentation, feature detection, texture modelling, and image matching. Most current mammographic image processing systems rely on techniques borrowed from other fields. For example, software used for detecting stars in the sky is converted to detecting small white dots in mammograms. Air-force technology based on neural nets and target recognition is transformed to be used for breast cancer recognition. Such approaches ignore the massive differences between the imaging systems and consequently lead to systems which work poorly, something which is unacceptable in this safety-critical application. In Highnam and Brady (1999), Chapter 1, a detailed clinical overview of the breast, breast cancer, and breast cancer screening using mammography is given, together with an account of the use of image processing in this context.

As noted in the previous section, an important goal of mammographic image analysis has been the detection of microcalcifications (Highnam and Brady, 1999, Chapter 11). Most of the many approaches have attempted to detect regions of elevated local contrast in the intrinsically noisy images. This approach has been largely unsuccessful and most authors have taken recourse to non-linear 
pattern classifiers such as neural networks in a (largely vain) attempt to improve the performance of the detection algorithm.

An alternative approach is to model the physics of image formation, including the mammogram-specific noise processes that degrade the image, and the X-ray scatter that blurs the image. The first successful algorithm of this type was by Karssemeijer (1993), an algorithm that has subsequently become the basis of the first commercial microcalcification detection system to be accorded FDA approval (Karssemeijer et al., 1998). The most comprehensive attempt to model mathematically the physics of mammogram image formation is the monograph Highnam and Brady (1999). This has led to novel mathematics and algorithms across the spectrum of clinical problems in breast cancer diagnosis, and has been termed physicsbased modelling (PBM).

\section{Physics-based Modelling (PBM)}

\section{The Physics of Mammographic Image Processing}

When a mammogram is performed, a beam of low energy $\mathrm{X}$-ray photons is directed towards a compressed breast. The intensity of the beam exiting the breast is related to the thickness and type of tissue, which attenuates the photons. The X-ray photons leaving the breast have to pass through an anti-scatter grid before reaching a phosphorous intensifying screen. If the X-ray photon is absorbed in the screen, light photons are emitted by the phosphor and these light photons expose a film, which is processed to produce a mammogram. The exposure to the breast is stopped once an automatic exposure control, positioned under a section of the breast, has received a set exposure.

This highly simplified description of the mammographic imaging process omits mention of a number of factors which significantly degrade the mammogram. These factors include: scattered radiation; "beam hardening" (a process, which causes the contrast in bright parts of the image to be reduced. The process occurs because low energy X-ray photons are differentially absorbed so that the beam energy rises (gets harder)); intensifying screen blur (the X-ray is absorbed in the intensifying screen and causes the screen to emit many light photons in all directions, these light photons expose the film over an area, not a precise point, and thus produce a blurring effect); and sometimes poor exposure due to the automatic exposure control being inappropriately located. A number of additional factors make it difficult to analyse mammograms quantitatively. These include variable processing results when developing films, system geometry, and the "anode heel effect". The latter refers to the considerable variation in radiation intensity throughout the volume of interest. Techniques to estimate and correct for it are outlined in Chapter 2 of Highnam and Brady (1999).

Digitisation further complicates the system. Mathematical models have been developed based on the underlying physical processes which attempt to minimise all of these factors, and the most important of these are summarised below.

\section{Mammographic Model}

Highnam and Brady's (1999) fundamental observation is that on the basis of the X-ray attenuation coefficients there consists only fat, "interesting" tissue (fibrous, glandular, cancerous), and calcification. Making an initial assumption that there is very little calcification present enables the breast to be considered as fat or interesting tissue. This, along with knowledge of certain calibration data, such as breast thickness, enables a mapping from any pixel within a mammogram to a quantitative measure of the breast tissue. This is termed the $h_{\text {int }}$, and its generation and subsequent use for many applications including cleaning up pictures and simulation is the basis of the PBM approach.

\section{The $h_{\text {int }}$ Representation}

The $h_{\text {int }}$ representation is a normalised representation of the mammogram, which is computer-generated from a digitised version of the mammographic image. For each column of breast tissue (that projects to a pixel) the program calculates the percentage of "interesting" tissue which leads to a novel surface representation for mammography, whose value at image location $(x, y)$ is $h_{\text {int }}(x, y)$. The importance of the representation is twofold. First, it enables normalisation of a mammogram by removing the variations due to changing imaging parameters and conditions such as time of exposure and placement of the automatic exposure control. Second, it encourages analysis of mammograms using the mathematics of surfaces rather than of images. For example, if a mammogram contains a cyst, then in the corresponding $h_{\text {int }}$ representation the cyst appears as a hill with a characteristic topography.

Quality control of breast screening centres dictates that most of the calibration data required to monitor the whole imaging process is routinely recorded. Using this data, the major challenges in generating $h_{\mathrm{int}}$ are, for analogue mammographic systems, collecting calibration data such as breast thickness, time-of-exposure, and film-screen parameters, and modelling the various degrading factors described above. The steps taken to generate $h_{\text {int }}$ are outlined below.

- Given the appropriate calibration data, the first step in generating $h_{\text {int }}$ is to convert the digitised image to one representing the energy imparted to the intensifying screen, as illustrated in Fig. 1. This involves knowing the relationship between pixel value in the image, $\mathrm{X}$ ray exposure and energy imparted which can be determined using calibration data derived from a stepwedge (see Highnam and Brady, 1999 for fuller details). 


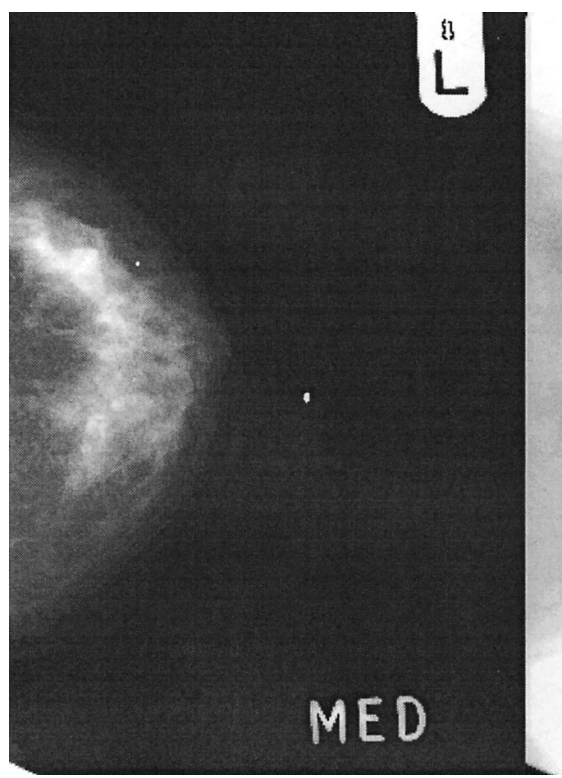

FIGURE 1 The original digitised image on the left is first converted to one which represents energy imparted to the screen shown on the right. See text for details.

- The intensifying screen blur is then modelled using established theoretical models of how a phosphor intensifying screen functions. This provides a point spread function specific to each screen, which can then be used in a deconvolution to remove the blur. This is illustrated in Fig. 2.

- The scatter component at a pixel is modelled as being closely related to the breast tissue in the surrounding neighbourhood. Using this model, together with results from numerous medical physics experiments, it is possible to derive a weighting mask, which describes the relationship between the neighbourhood and the scatter component in the centre of the neighbourhood. Fig. 3 shows an original mammogram, an energy imparted version and the corresponding scatter component.

- Extra-focal radiation is known to be a problem around the edge of the breast. Models of the expected curve of the breast edge are derived in order to estimate extrafocal radiation penetration. The models are based upon

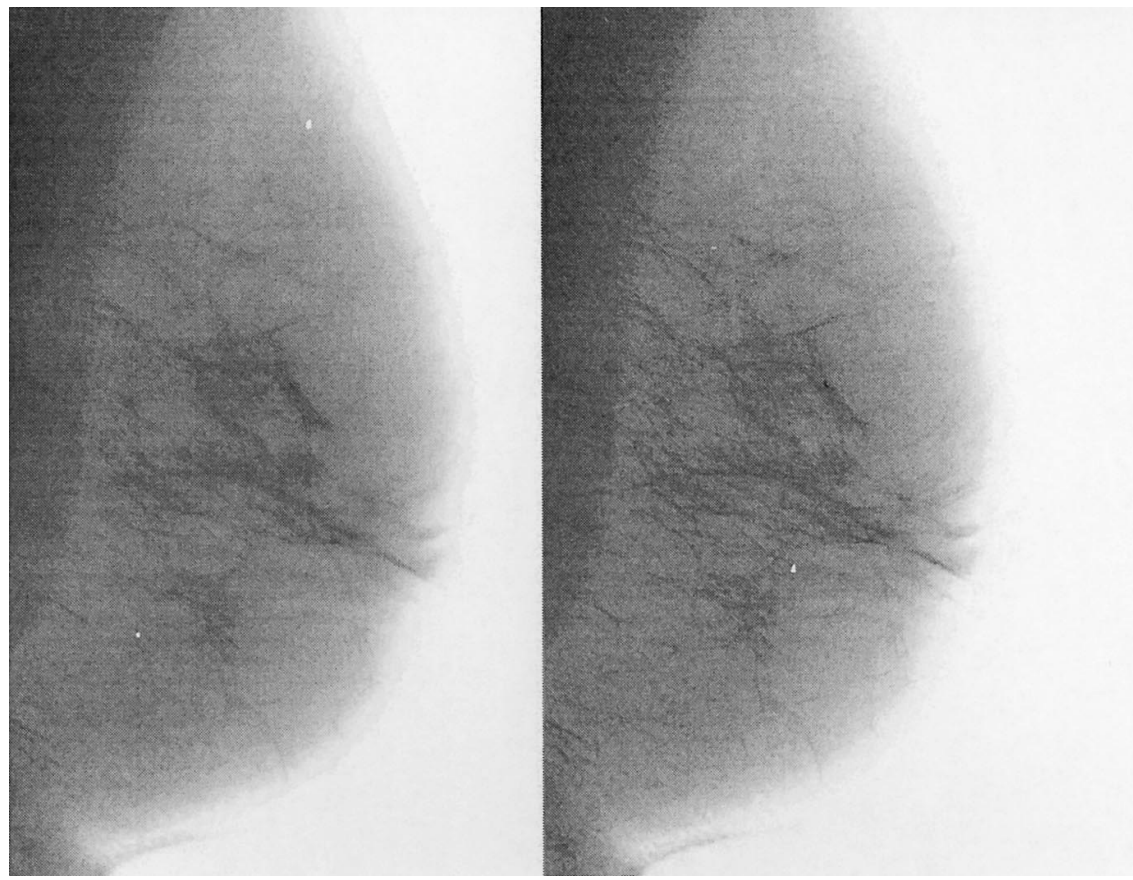

FIGURE 2 The image on the left shows the original energy imparted image, whilst the one on the right shows the blur removed image. See text and (Highnam and Brady, 1999) for further details. 


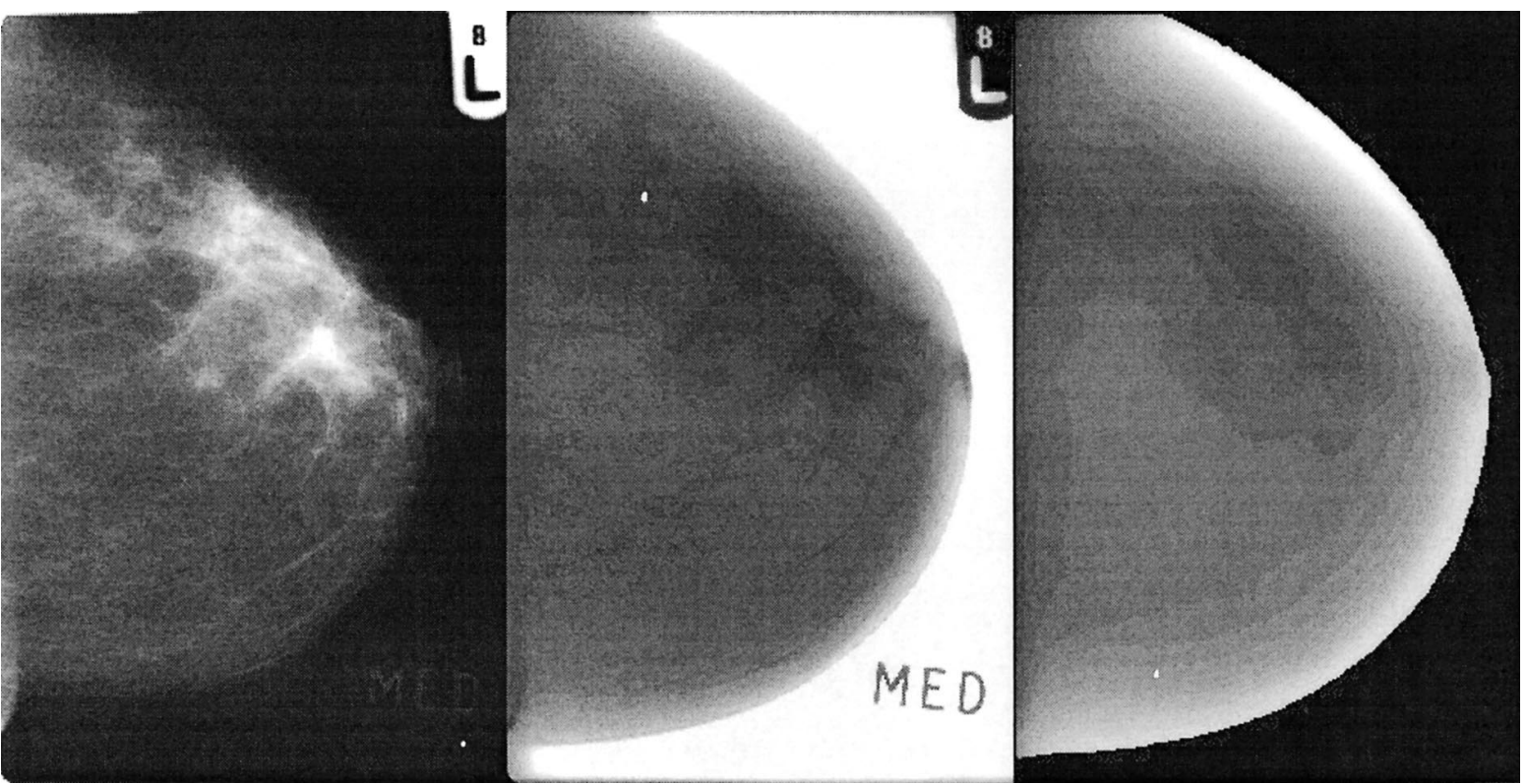

FIGURE 3 An original mammogram, an energy imparted version, and the corresponding scatter component.

assumed knowledge of the breast and knowledge of its constituents in certain areas.

The end result of this processing is a floating point image where the pixel values have been converted to values representing $h_{\text {int. }}$. These values are quantitative and objective values, independent of the imaging system itself. The most obvious way for an engineer to represent this is as a surface, as shown in Fig. 4. However, radiologists have no experience of diagnosing from surfaces and thus in order to show the images to radiologists the $h_{\text {int }}$ values must first be converted back to appear like an ideal mammogram. This improved image then becomes the basis for a wide range of image enhancement techniques.

\section{Image Enhancement}

Because of the number of degrading factors involved in the mammographic imaging process, the final image is relatively poor compared to what an ideal image should

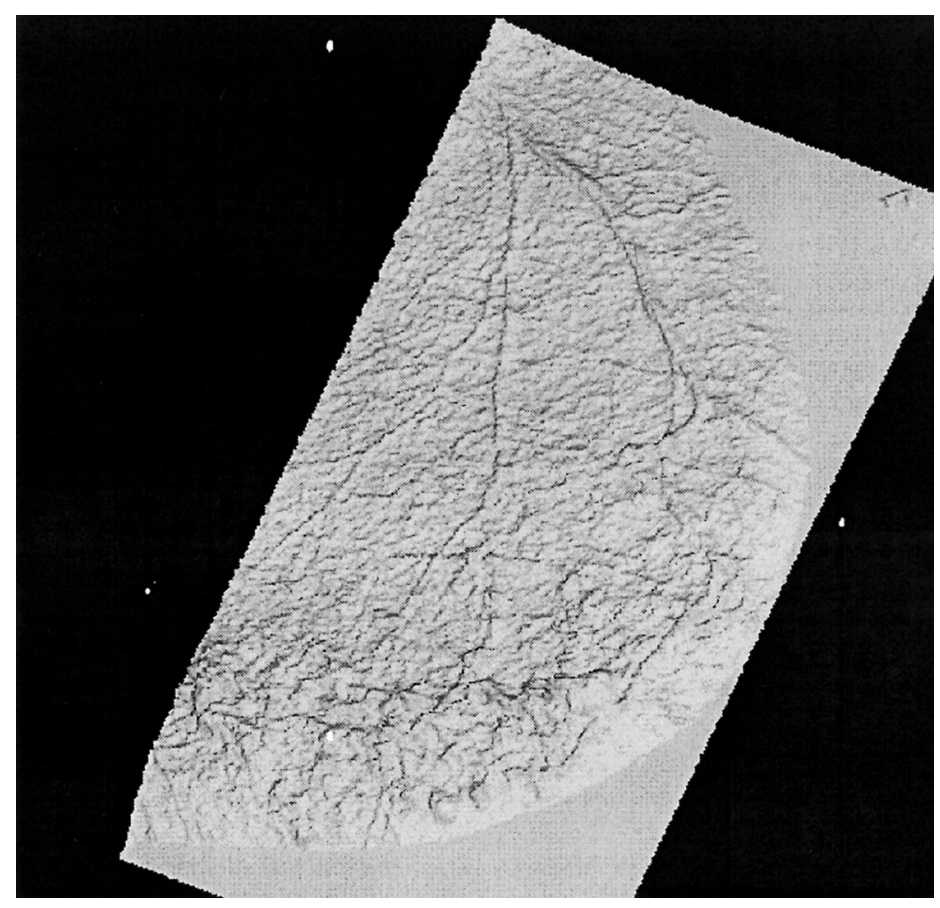

FIGURE 4 An example of the $h_{\text {int }}$ representation. 


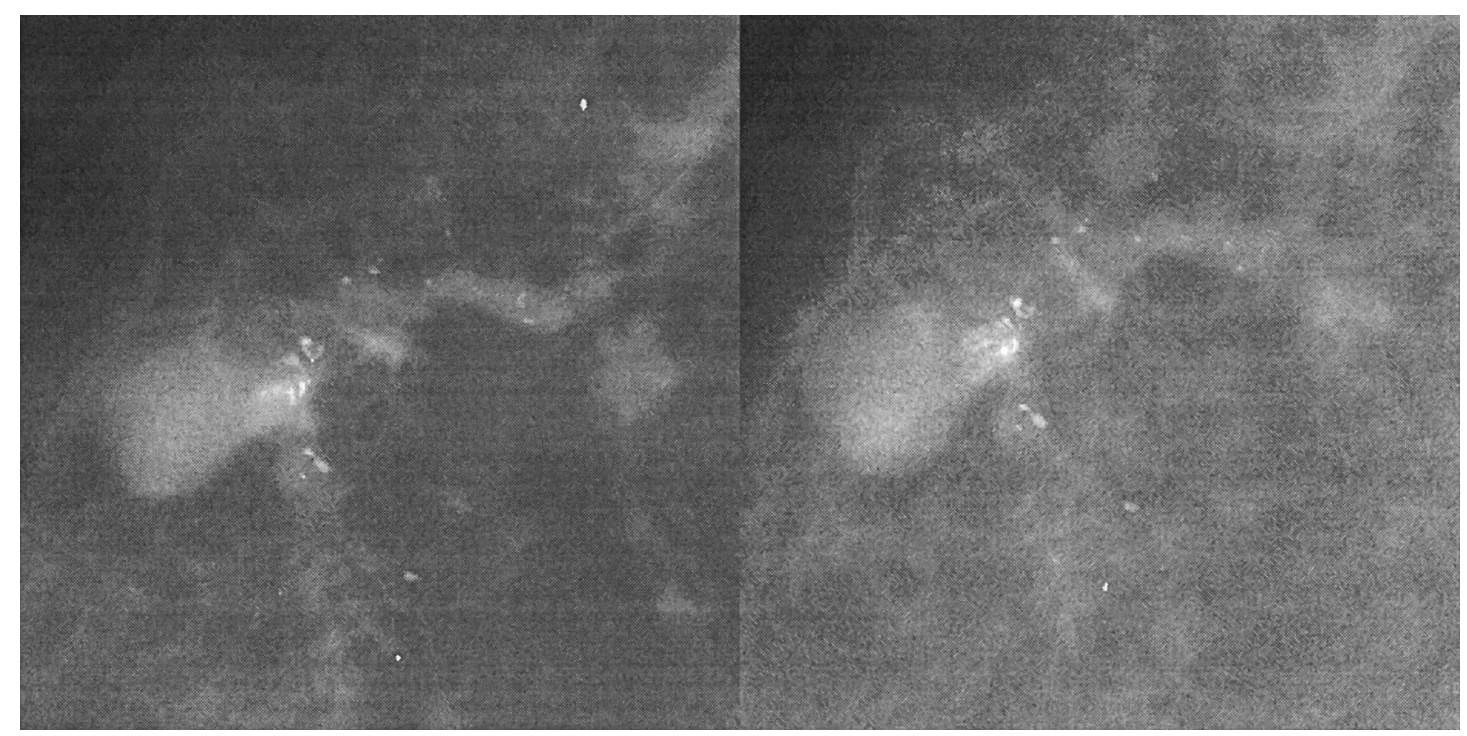

FIGURE 5 Image enhancement by modelling of scattered radiation. The left image shows a section from a mammogram taken with an anti-scatter grid, whilst the right image shows the same area but taken without a grid and then digitally enhanced.

be. Our modelling of the degrading factors allows us to remove their effects and thus guarantee a cleaner, easier to diagnose image. The use of models rather than conventional ad hoc algorithms make it less likely that the processing will introduce any artifacts or remove any important signs: vitally important in a safety-critical application such as mammography. A classic example is of a tumour blending in to the background due to some of the processes outlined above. An "edge enhancement" button might well sharpen up the tumour's appearance to make it look benign rather than malignant.

\section{Reducing the Radiation Dose}

Modelling scattered radiation, as outlined above, enables a soft removal of its effects rather than using a hard solution, namely an anti-scatter grid which requires a doubling of the radiation dose. Fig. 5 shows on the left a section from a mammogram taken with a grid and on the right the same area but taken without a grid and then digitally enhanced.

\section{Detecting Microcalcifications}

It will be recalled that the detection of microcalcifications has been a major goal of mammographic screening and image analysis. Microcalcifications are quite often benign, for example, they may be small deposits of calcium in blood vessels. The detection and the characterisation of microcalcifications as benign or malignant continues to be a major open problem, though there has lately been some encouraging progress. Microcalcifications rarely exist singly, and it is widely considered that the position, shape,

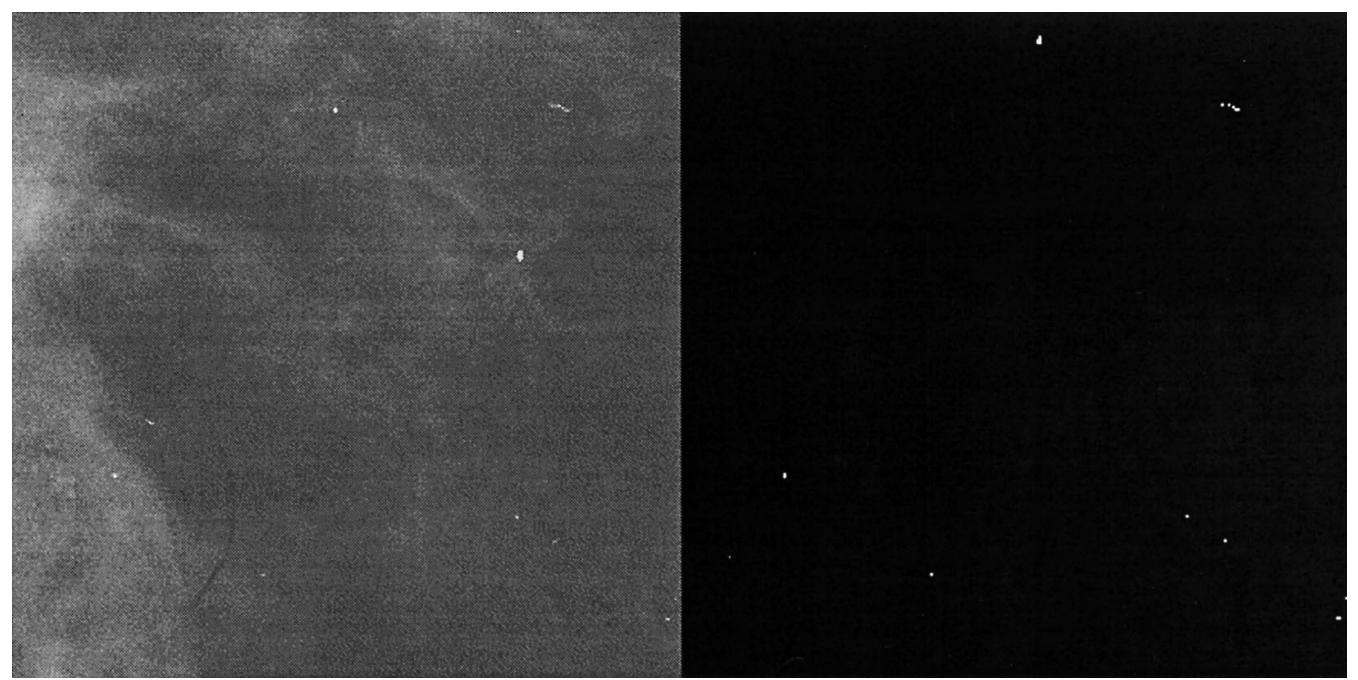

FIGURE 6 Removal of shot noise: the section on the left is an original mammogram with shot noise, i.e. dust and dirt, and on the right is the detected noise shown as tiny white dots. 
and statistics of a cluster of microcalcifications contributes to the characterisation of the cluster. Recall however that the breast is tightly compressed to different extents in the $\mathrm{CC}$ and MLO views. This means that the statistics of a cluster estimated in either view confound both the compression and the projection of mammography.

There are four stages to this work, all based on and derived from our model of the imaging process. The first stage involves suppressing radiographic noise with appropriate model-based image processing, the second stage involves removing shot-noise due to dust and dirt and the third stage involves the identification of individual microcalcifications. The final stage detects clusters of microcalcifications.

The removal of shot noise is illustrated in Fig. 6. This algorithm, based directly on the physics of the imaging process, detects $97 \%$ of all shot noise and does not remove any microcalcifications. The algorithm works by determining those points which cannot possibly have been present throughout the imaging process since they are too sharp. The maximum degree of sharpness can be determined using the physics models of the blurring functions present (Highnam et al., 1999).

The microcalcification detection algorithm is based upon a quantitative assessment of the size and contrast of the suspect regions. The fundamental assumption underlying the generation of the $h_{\text {int }}$ representation is that the breast consists of only fat and interesting tissue, which is reasonable since pixels corresponding to calcifications make up only a fraction of a percent of the total number of pixels. The X-ray attenuation coefficient of a calcification is typically 26 times that of interesting or fatty tissue, and is therefore equivalent to the attenuation through a much thicker layer of interesting tissue. Calcifications can therefore be detected by considering the ratio of interesting tissue volume, computed from the $h_{\text {int }}$ surface, to the total volume of a localised region of breast tissue computed from its spatial dimensions and an appropriate three-dimensional model. This ratio will be high for calcifications. Further details of this method can be found in Highnam and Brady (1999), where the technique is shown to be working at near $97 \%$ detection of individual microcalcifications with small numbers of false positives. This process is illustrated in Fig. 7. On a small dataset, this algorithm is currently returning very low false positives and about 98\% detection of true positive clusters.

\section{Matching Between Views}

It is often very difficult for a radiologist to observe an object in both cranio-caudal and medio-lateral oblique views of the same breast. The key step in aiding this task is to model the effects of breast compression. We have developed models which allow a marked point in one image (the round circle on the left in Fig. 9) to be matched to a curve of likely position in the other view (the white curve on the right in Fig. 9). The six stages of the algorithm to combine a cranio-caudal and mediolateral oblique image to form a representation of the 3D uncompressed breast are outlined in Fig. 8.

Our method, which is based on assumptions about certain reference points and tissue movement and elasticity, works by mapping from a point $\mathrm{A}$ to the three-dimensional line along which that point must lie. The three-dimensional line, in the compressed breast, is then uncompressed using our mechanical model of the breast. It is then rotated to be in the other view and "compressed". Projection down onto the two-dimensional plane gives the corresponding line for the point. Our method is typically $2 \mathrm{~mm}$ closer than the radiologists'.

The straight lines indicate where trained radiologists expect the mass to be. The methods that we have developed (Yam and Brady, 1999) perform significantly better on average than experienced radiologists.

The use of a model allows us to map to three-dimensional, if an object's position in both the two-dimensions views is known. This can also be applied to microcalcifications, where two views of the same cluster can be matched and combined to provide a three-dimensional representation.

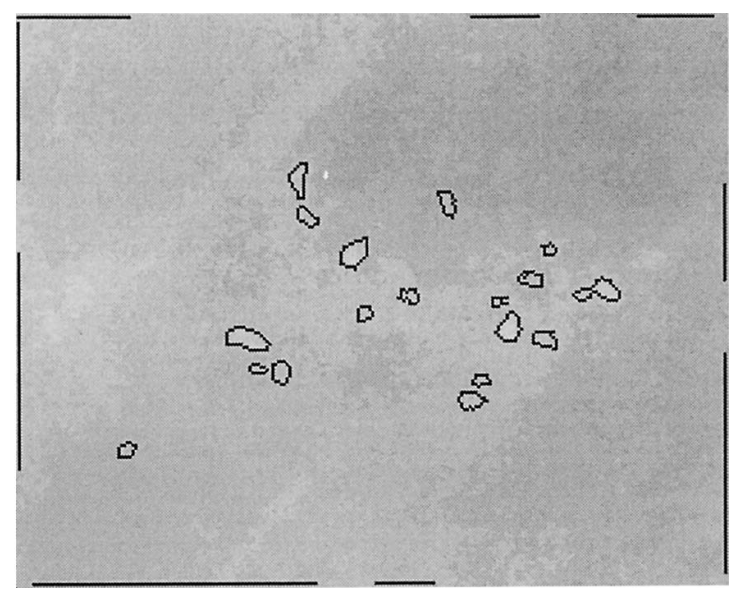

FIGURE 7 Detection of microcalcifications: the left panel shows an image which has had the shot noise removed; the right panel shows the results of the detection algorithm. 


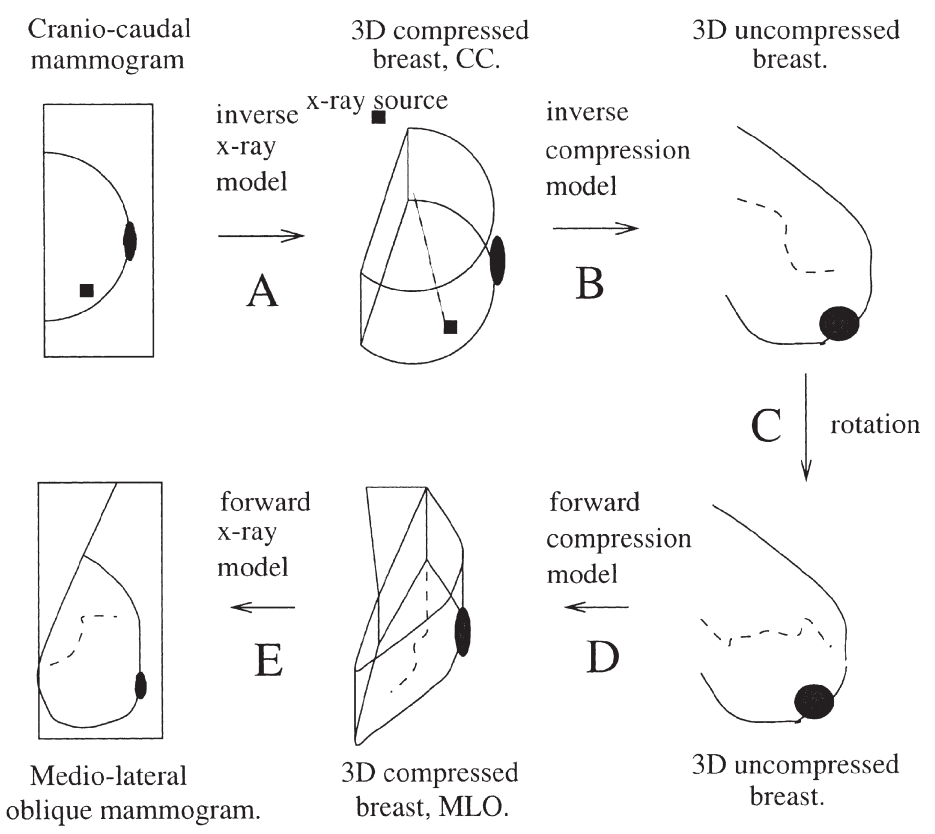

FIGURE 8 The six stages of the algorithm to combine a cranio-caudal and mediolateral oblique image to form a representation of the 3D uncompressed breast.

Statistics computed from the three-dimensional representation should be more robust in differentiating between benign and malignant microcalcifications than their twodimensional counter-parts.

\section{Detecting and Removing CLS}

Curvi-linear structures (CLS) in mammograms arise for a variety of reasons including fibrous strands, blood vessels and the lymphatic system. Apart from their intrinsic interest, the CLS greatly complicate the visual appearance of a mammogram and make the detection of masses (benign or malignant) more difficult.
Several approaches have been pursued to detect the CLS in mammograms. First, conventional feature detectors do not give good results, largely because the elevation in intensity at points perceived as belonging to a CLS is often slight, and the whole image region is noisy. Instead, we model CLS both spatially (local linear structures) and in 3D as being structures of elliptic profile full of interesting (non-fat) tissue (Highnam and Brady, 1999; Cerneaz, 1994). Then, by modelling the image formation process, the expected visual appearance of a CLS is derived. The model is then used to detect such CLS. This algorithm, developed by Cerneaz (1994), works only on a single spatial scale, whereas CLS occur at a wide

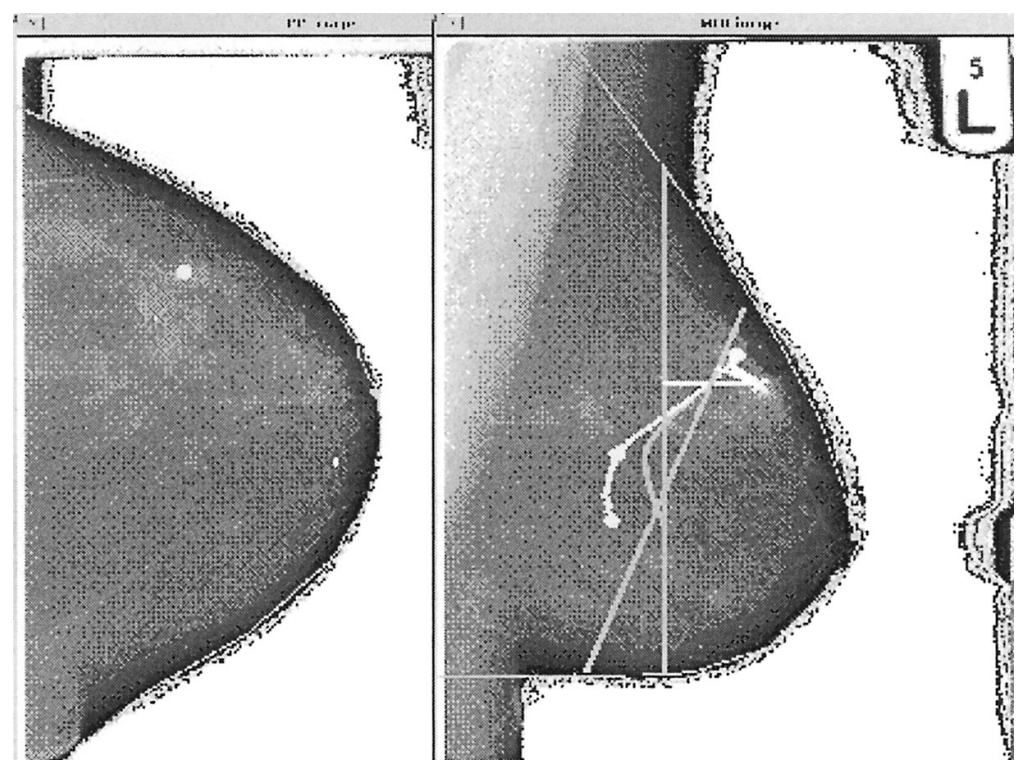

FIGURE 9 Tracing the movement of objects in moving from the cranio-caudal to the medio-lateral oblique views of the same breast. 


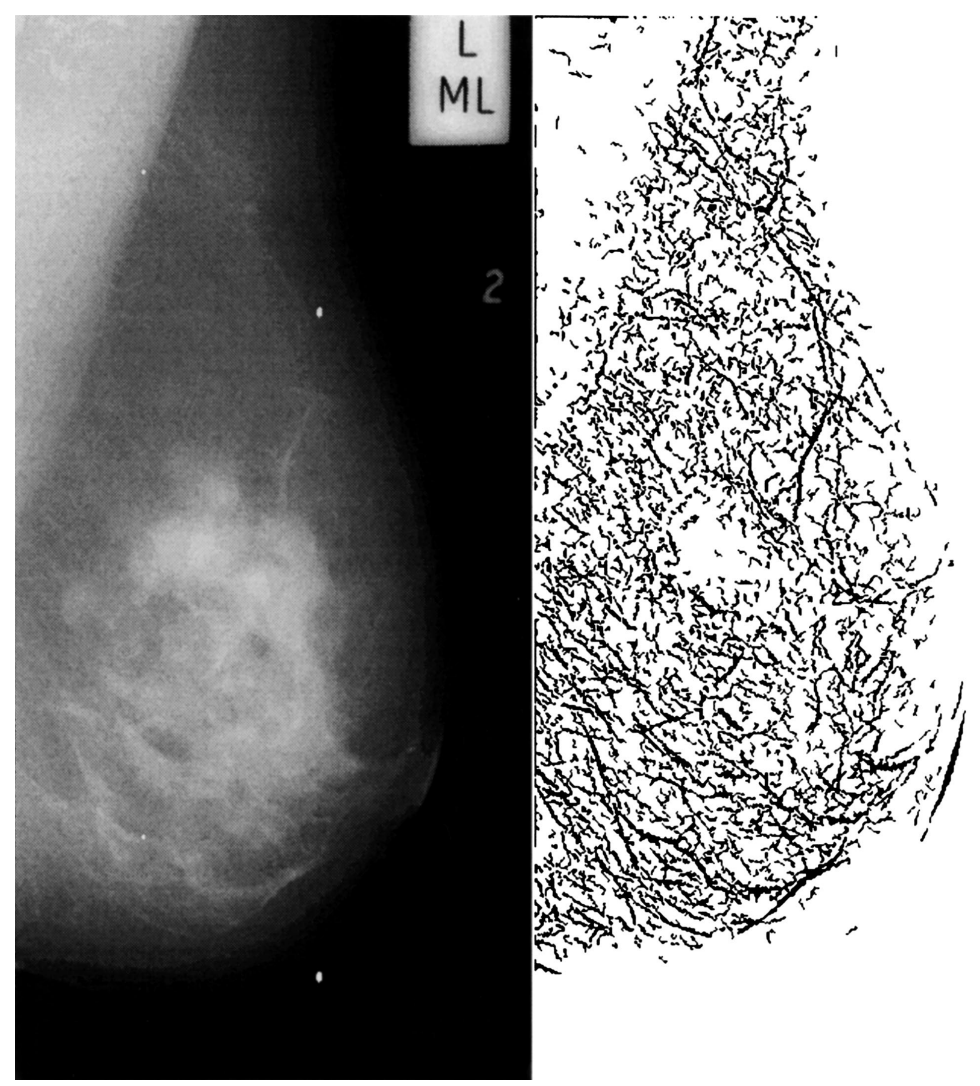

FIGURE 10 Removing CLS: the modelling allows the structures shown in the right image to be determined from the original mammogram shown on the left.

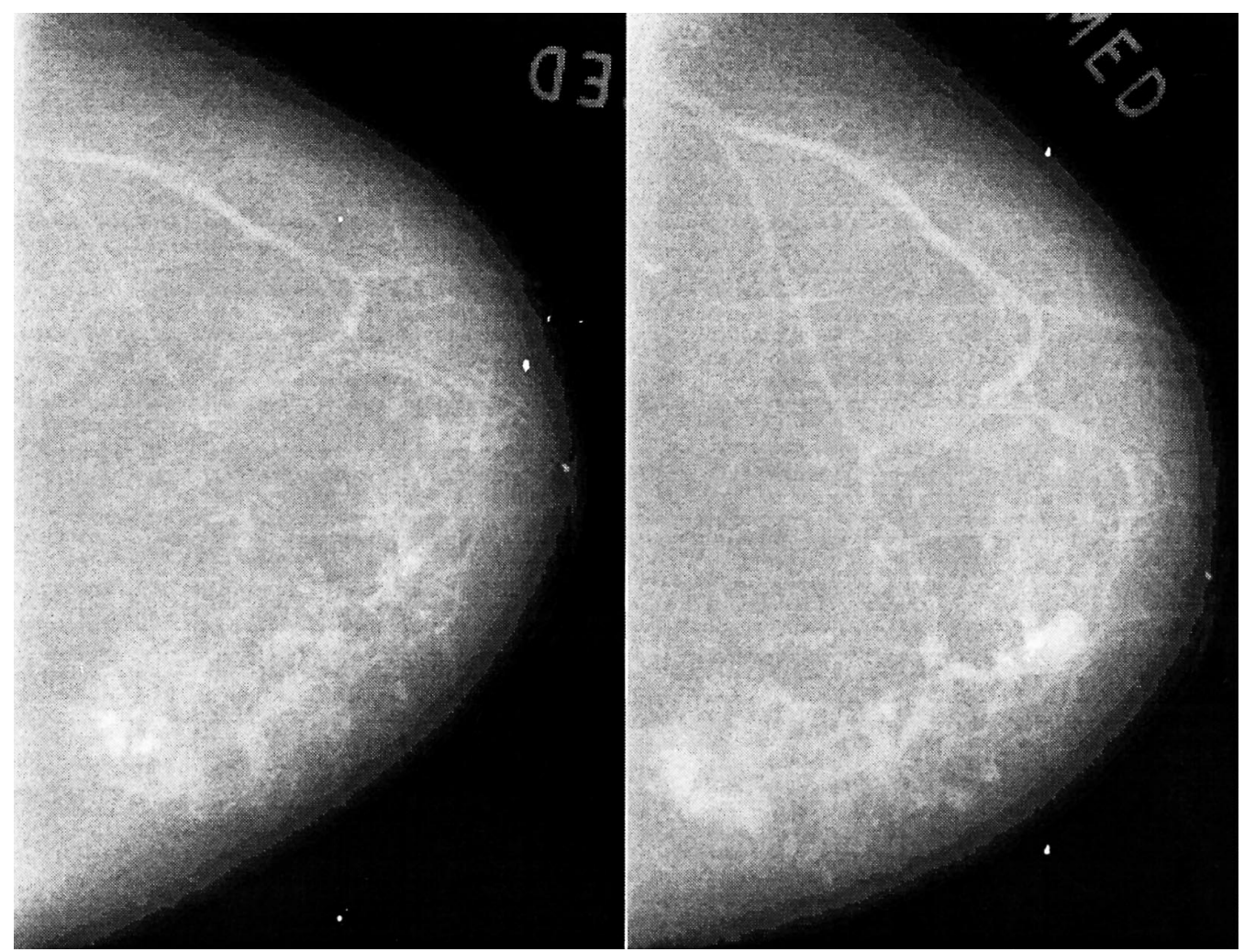

FIGURE 11 The image on the left was taken in 1992, whilst that on the right is from 1996. 


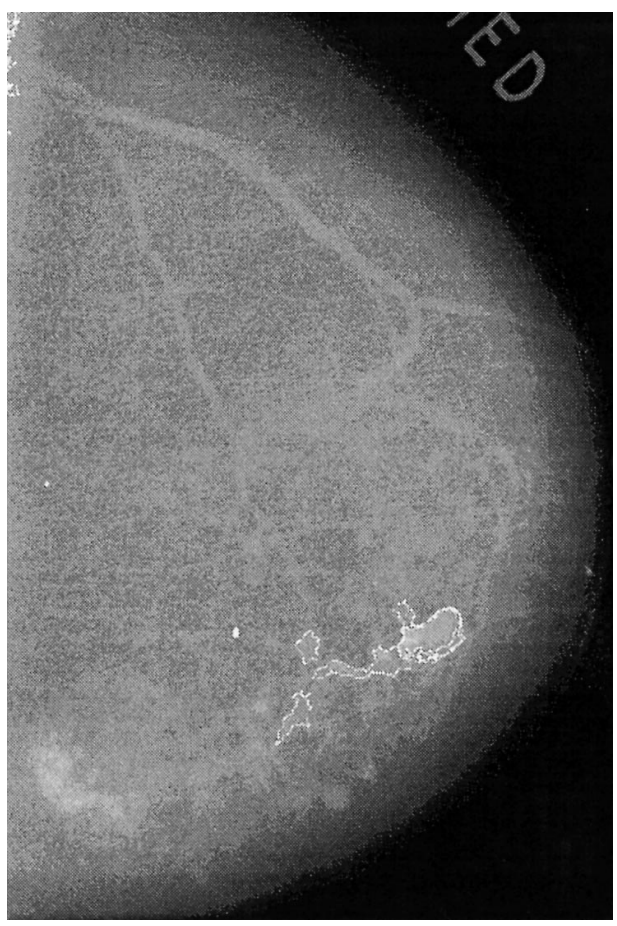

FIGURE 12 The results of the matching algorithm applied to the images shown in Figure 11. The marked region is cancerous.

range of spatial scales in mammograms. More recent work (Zwiggelaar et al., 1996) attempts to detect CLS using a set of "modal variations" determined by principal components analysis on a training set of idealised cases of CLS. Current work aims to apply recently developed techniques for feature detection, based largely on the observed phase congruency of features. Fig. 10 illustrates this method and shows an original mammogram and the curvilinear structures determined by this process.

\section{Detecting Masses}

Detecting masses on the basis of matching temporal and bilateral mammograms can be done by building up a representation of each breast which accurately reflects the internal anatomy (Kok-Wiles et al., 1998). Assumptions about compression and movement over time are then used to match those structures. An alternative approach that is being developed is to warp one outline onto the other outline and to use internal land-marks to aid that process. In Figs. 11 and 12 this is illustrated for mammograms taken 4 years apart. In this example, a mass is detected on the basis of there being no corresponding match in the earlier mammogram: the marked region in Fig. 12 is indicative of a newly formed mass, in this case cancer.

The work in Marias et al. (2000) has resulted in a robust registration framework for comparing temporal mammograms. This non-rigid system uses a two-step approach for firstly deforming the images using a curvature analysis of the breast boundary and secondly, utilising internal landmarks to complete the complex internal deformation of the breast, typically due to the slightly different compression characteristics of successive mammograms. A good example of this process is shown in Fig. 13 where
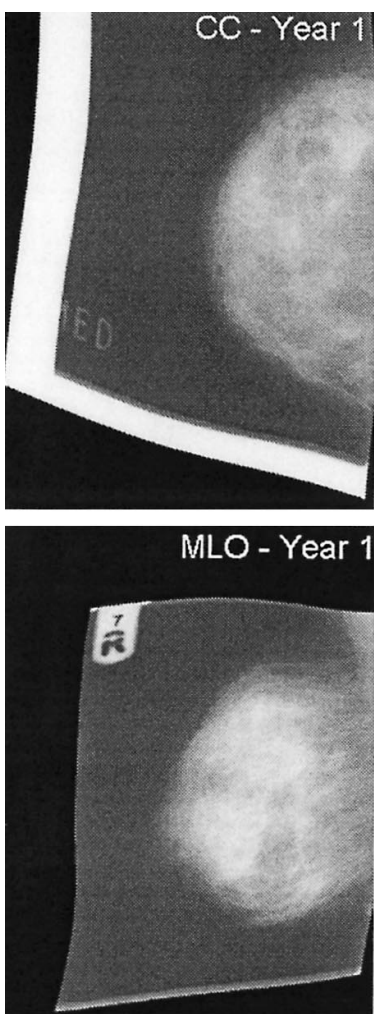
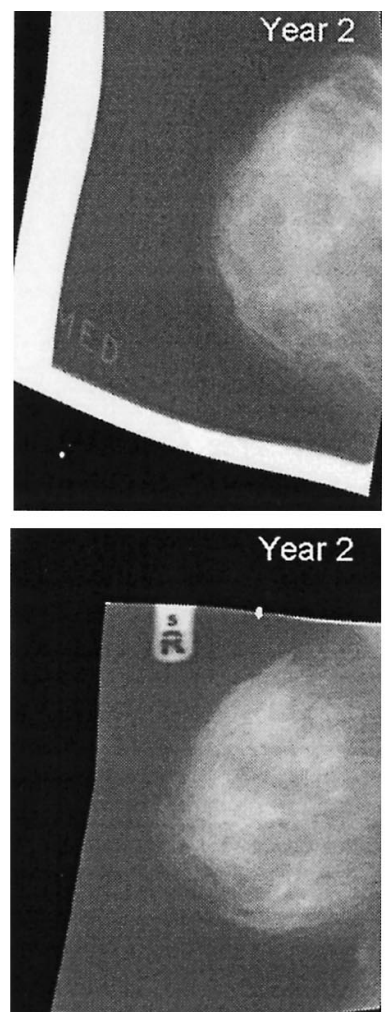
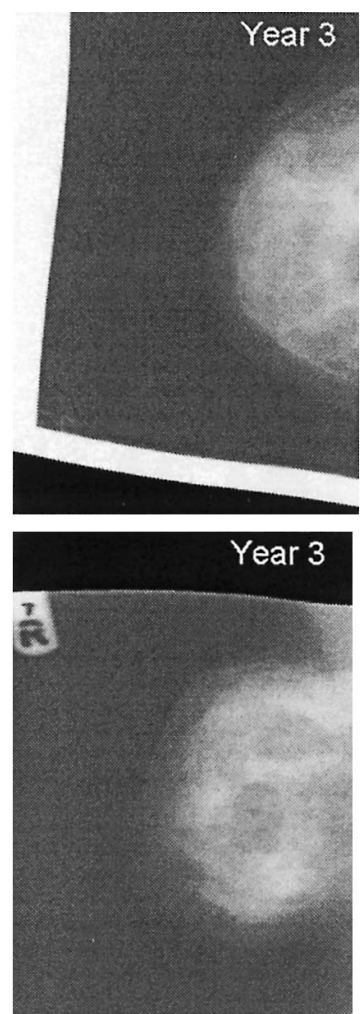
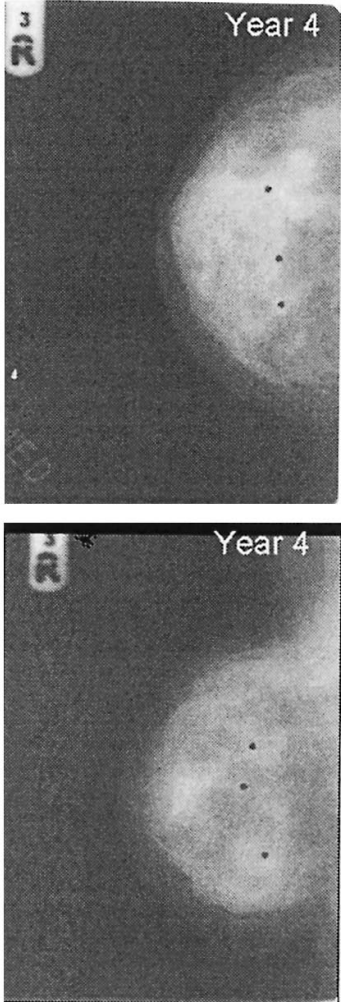

FIGURE 13 Registration of temporal mammograms for screening, postoperative assessment and pathology comparison. 
a patient has had 4 years of successive mammograms where in year 4, some coarse calcifications indicative of DCIS (ductal carcinoma in-situ) were observed. This is a particularly interesting case as the patient had previously undergone an upper-quadrant mastectomy to remove an earlier lesion and it was uncertain whether or not the calcifications were due to surgical scarring or an active lesion.

This elastic matching of temporal mammograms extends very naturally to screening, where compensation for changes in compression and geometrical normalisation enables much better comparison between changes in breast tissue. This has been shown to be applicable to interval cancers and the evaluation in local changes due to HRT (Marias et al., 2000). Fig. 14 shows a typical example where an interval cancer has appeared in a patient screening and where registration has enabled a retrospective evaluation of the tumour pathology (and consequentially the clinical decision making).

\section{Combining Information from Mammography and MRI}

Although screening mammography is critical for early cancer detection and has been shown to have significantly affected mortality statistics in women (Kok-Wiles et al., 1998), mammography is not particularly useful for diagnosis in young women, women with dense breast tissue (perhaps due to HRT) and women who have already had surgical intervention. In these cases, other modalities such as contrast-enhanced MRI and ultrasound have an important role in understanding the pathology and tissue characteristics.

In the case of DCIS, the typical indicator in mammography is coarse, highly localised clusters of microcalcifications. The work by Highnam et al. (1996) with respect to $h_{\text {int }}$ normalisation makes the detection of the calcifications quite robust. However in younger women, or women with dense breast tissue, it is difficult to differentiate the extent of the pathology from the rest of the parenchyma. Contrast-enhanced MRI provides complementary information about the tumour by enhancing the angiogenesis of the breast pathology. The use of a pharmacokinetic model (Kaiser, 1990; Tofts and Kermode, 1991; Hoffman et al., 1995; Heyton et al., 1997) to segment the cancer has been shown to be diagnostically useful (Behrenbruch et al., 2000). Such pharmacokinetic techniques are based on a model of gadolinium chelate uptake and exchange between blood plasma and the tissue extracellular space. By parameterising the contrast enhancement with such a model, it is possible to segment enhancing regions on the basis of the rate and amplitude of contrast uptake. The problem with DCIS is that it typically does not enhance well with MRI (due to the lack of extensive blood supply other than from the ductal tissue) and therefore is often missed in an MRI examination.

Our current research (Behrenbruch et al., 2000) involves using a projection of the contrast enhancement information and non-rigid registration to match the geometry of the two mammogram views (CC and MLO) to the geometry of the MRI acquisition. This data fusion approach enables indicators such as calcifications to be resolved in the reference frame of the MRI volume. This enables the radiologist to detect better subtle enhancement through knowing the location of calcifications in threedimensional. This three-dimensional reconstruction approach is complementary to the work in Yam and Brady (1999) although it provides additional information by fusion of the structural representation in the mammogram with the functional representation of MRI. An example of this is shown in Fig. 15a where three clusters of calcifications are seen in the two mammogram views and have been reconstructed in the geometrical reference frame of the MRI scan. It was then possible for the clinician to look at a specific region of the MRI scan for subtle enhancement (shown in Fig. 15b).
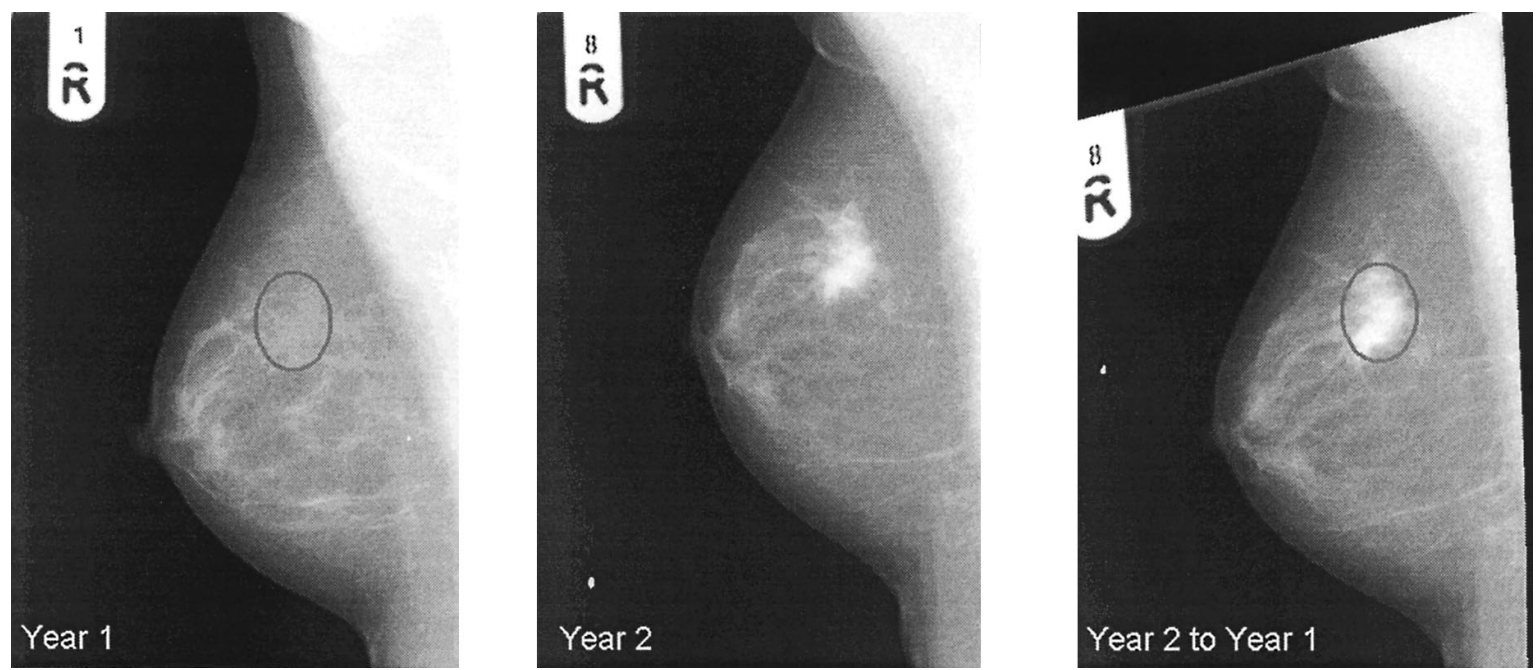

FIGURE 14 An interval cancer compared using non-rigid registration of temporal mammograms. The interval cancer was actually present (but undetected) in the first mammogram. 

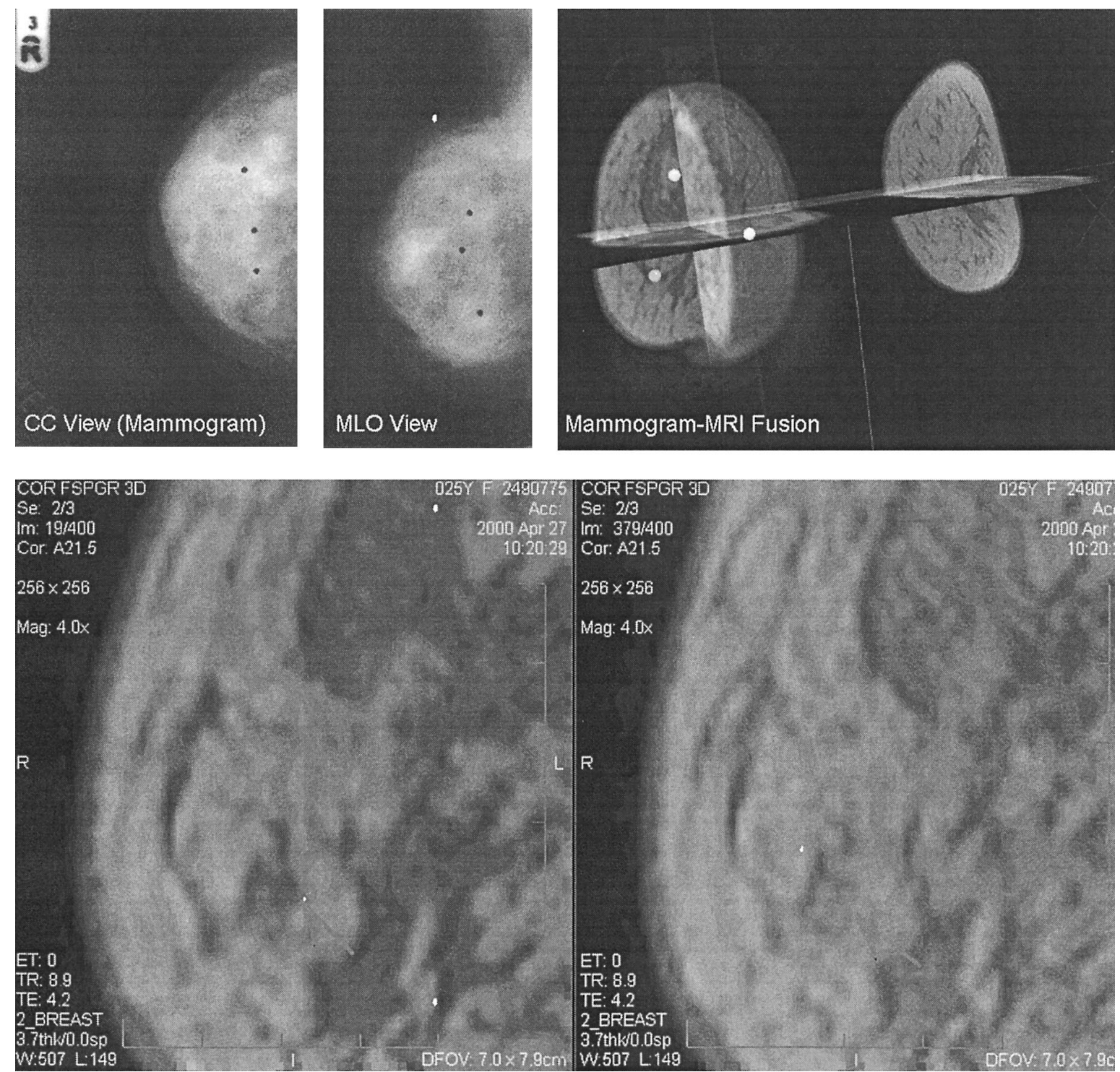

FIGURE 15 (a) An example of data fusion, showing the location of calcifications in the geometrical reference frame of the MRI volume. (b) The corresponding pre- and post- contrast enhancement of a suspicious calcification cluster (fusion with mammography).

The comparison of pathological indicators is not restricted to DCIS, however. In terms of understanding the relationship between the imaged structure of a tumour and the functional characteristics, X-ray/MRI data fusion provides complementary information for pathology assessment (Behrenbruch et al., 2000). An example is shown in Fig. 16, where a large, poorly differentiated carcinoma appears in the mammogram as a dense, speculated mass. The clinical response to such a mass would typically be a localised excision as it is a large, but relatively contained pathology with very little connective vascularity. However, analysis with the MRI "functional" representation shows that in fact the tumour has two foci, indicating a more invasive grade of cancer which may have a different treatment requirement.

Data fusion is likely to be an important aspect of future work involving modelling of the growth characteristics of cancer, particularly if correspondence between dynamic imaging techniques and the results of drug treatment regimes can be understood. Current research involves studying the effects of neo-adjuvant chemotherapy on the pharmacokinetic enhancement of breast MRI (Moore et al., 2001). By using non-rigid registration combined with robust segmentation algorithms (that once again use physics-based models to perform $\mathrm{T} 1$ correction of gadolinium enhancement (Armitage and Brady, 2000)) we have been able to quantify the change in angiogenesis of a tumour within the framework of oncological models for tumour proliferation. In the future, this research may form the basis of an inverse problem that may provide a better understanding of cancer growth. Fig. 17 shows an example of a visualised change in vascularity due to neoadjuvant chemotherapy.

\section{PBM in Simulation and Teaching}

The modelling of the imaging process provides all the information necessary to teach radiographers. Much more 

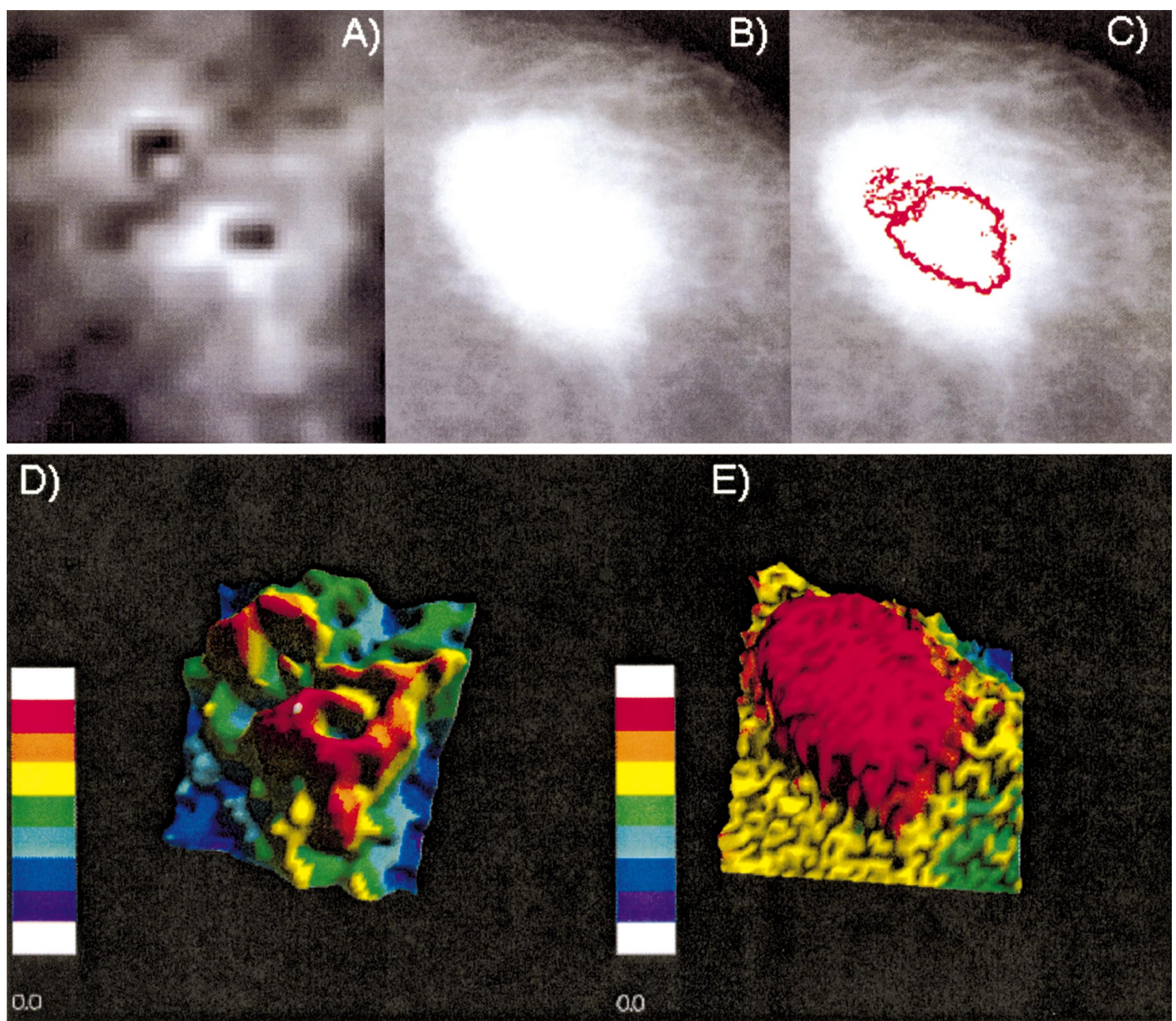

FIGURE 16 Structural vs. "functional" comparison of pathology through mammography-MRI data fusion. (A) shows the projection of the contrastenhanced MRI volume, clearly showing two necrotic centres; (B) shows the same tumour in the mammogram. It is worth noting (via C) that some thresholding and edge detection of B also indicates multifocal growth. (D) and (E) show surface representations of the MRI enhancement and the mammogram, respectively. The colour map is simply for improved visualisation (i.e. relative height is colour coded).

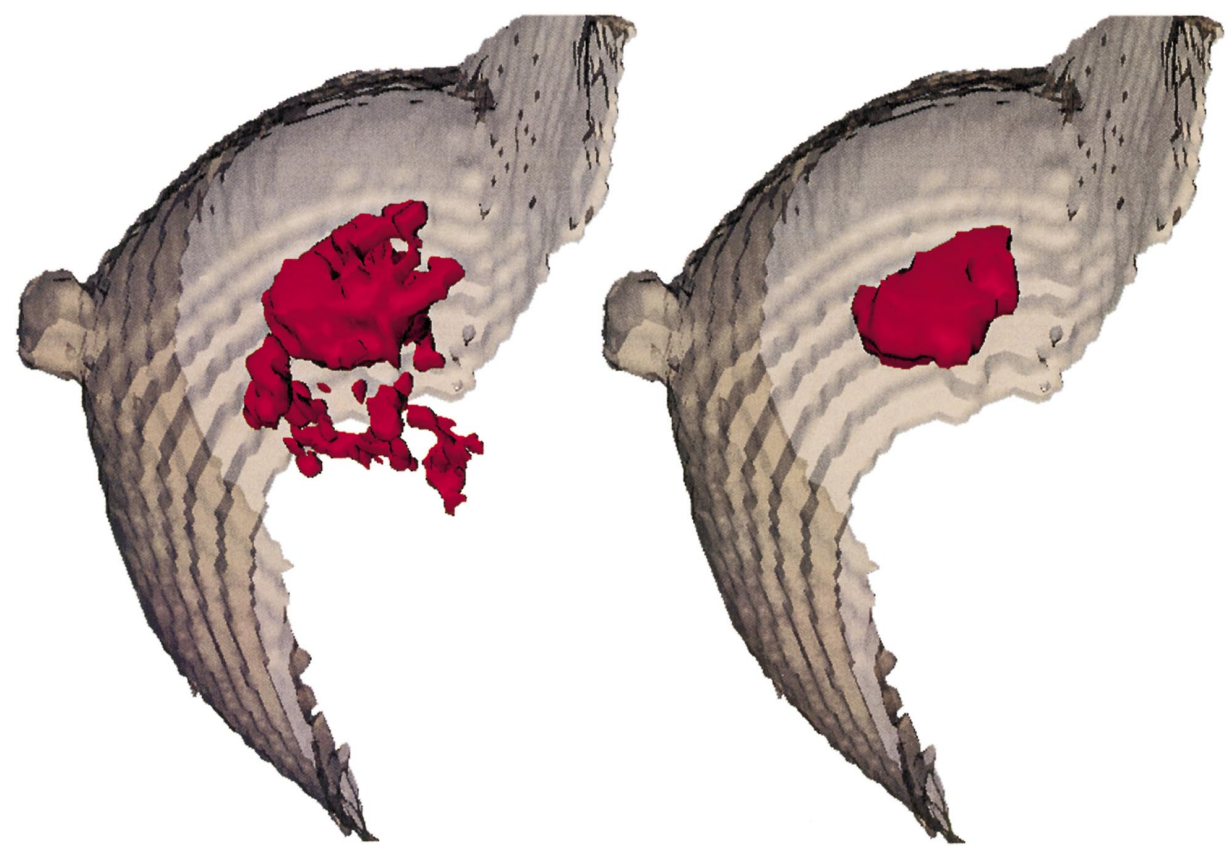

FIGURE 17 Registration, physics-based modelling and image processing for assessing the change in tumour vascularity (growth) due to neo-adjuvant chemotherapy. 
powerful though, is the ability to add on to the $h_{\text {int }}$ surface objects representing breast cancer. An example might be a sphere filled with non-fat tissue. After addition to the $h_{\text {int }}$ surface, the X-ray process can be simulated to show how that object might appear. In this way, cancers can be added to many previously normal mammograms and used to monitor radiologists' performance and help teach, "what would it look like if there was a mass just there?". This can be advanced to become a diagnostic tool: "this mass when added to the breast looks like the mass which is actually present, therefore I know the properties of the present mass and can use those parameters to help me diagnose". However, accurate simulation requires parameters relating to tumour growth, tumour shapes, and how tumours deform under compression.

\section{ENHANCEMENT OF IMAGE PROCESSING THROUGH MATHEMATICAL MODELS}

In the above reviews we have given a brief outline of recent developments in the mathematical modelling of cancer development, and of recent advances in breast mammography using PBM. As we suggested in the Introduction, the information gained from this approach to image analysis can be incorporated into existing models to give improved mathematical descriptions of the physiology, which can then in turn be used to help overcome some of the current challenges in image processing and hence improve the detection of cancer.

\section{Current Challenges in Image Processing}

The appearance of a mass in an intensity image depends strongly on the compression of the breast. Benign masses tend to have sharper boundaries, whereas malignant masses blend more gradually into their surroundings. Since it is not obvious how to combine a number of such features into a decision, most research involves training neural networks. The best (clinical) performance reported to date is about $70 \%$ correctly labelled masses, that is to say it could not be relied upon for three women in 10 presenting with a lump! It is therefore important to study the ways in which mathematical models might be used to suggest features that "characterise" a mass and that are stable/invariant under small changes in compression.

Increasingly, radiologists take both a cranio-caudal (CC) and a medio-lateral oblique (MLO) mammogram, typically angularly separated by $60^{\circ}$. However, the problem is substantially more complicated than the wide-angle stereovision problem because the breast is compressed to a significantly different extent in the two views. This problem necessitates the incorporation of more realistic mathematical models into the development of better algorithms for matching a CC to a MLO mammogram. This would open the way to the solution of two important problems:

- microcalcification clusters could be viewed as threedimensional constellations. The overlapping projections of those clusters in a mammogram could be separated. Spatial (three-dimensional) statistics could be derived for each cluster; statistics of projected clusters are misleading. The spatial structure of a cluster is conjectured by radiologists to be an important indicator of malignancy. For example, if there are many microcalcifications within a $1 \mathrm{~cm}$ cube then that cluster is more likely to be malignant.

- There has been debate among radiologists about the existence or otherwise of a "halo of safety" surrounding a mass in a mammogram, and, if it exists, whether it is relevant to a diagnosis of malignancy. Highnam et al. (1996) developed the first quantitative measure for such a halo, and presented evidence that if its size is consistent between an MLO and a CC mammogram then it is strongly suggestive of malignancy. If this conjecture turns out, after rigorous experimentation, to be correct, then a CC-MLO algorithm would give additional important information to characterise the mass.

\section{Improving the Mathematical Models}

\section{Development of a Model of Breast Compression}

The above points illustrate the importance of developing a sequence of increasingly realistic models of breast deformation under compression, and of incorporating them into image analysis algorithms to improve on those developed to date. Mathematical modelling of soft-tissue deformation is an area of much current research, with primary application areas being in medical simulation (e.g. surgical simulators), whole-heart modelling, soft connective tissue and solid tumour modelling (e.g. cartilage and intervertebral discs) (Fung, 1993; Skalak et al., 1996; Byrne and Chaplain, 1996b; Netti et al., 1997; Delingette, 1998; McCulloch et al., 1998). The mathematical approach taken is largely determined by the application: in surgical simulation, of primary importance is speed of computation, and linear elasticity theory is commonly used; in whole heart, and for models of breast deformation, modelling accuracy of deformation is paramount and the much more complex finite deformation elasticity theory must be used. A major problem arising in modelling the deformation of the breast is the number of biological structures that are present, each with different elastomechanical properties, with each giving varying constitutive laws throughout the breast.

The constitutive laws required in developing such models depend crucially on the materials that are being deformed. The geometry of the breast is also important, 
and basic breast anatomy can now be determined from digitised MRI images. A three-dimensional finite element model for a particular heterogeneous breast must incorporate a realistic representation of the threedimensional domain incorporating descriptions of the spatial arrangements and heterogeneous mechanical properties of the different tissues. This may be developed from the results of matching the CC-MLO mammograms, or from contrast-enhanced MRI data. The influence of age on tissue must also be considered.

\section{Development of a Model of Tumour Growth in Spatially Heterogeneous Tissue}

A mathematical model such as that described above would provide insight into the effects on anatomical structures of physical compression from different directions, the way that abnormal (both malignant and benign) structures interact with the local tissues; and the tissue composition of anatomical structures. The information is now available to provide a detailed map of the spatial heterogeneity of breast tissue within specific breasts. Using the mathematical modelling techniques outlined in "Previous mathematical models", it should therefore be possible to build a mathematical model of cancer growth from its initial inception right through to metastasis. Such models would build on existing work on tissue growth due to internal dynamics (Dillon, 2001). By "growing" a breast-specific cancer in a breast in this way, and by using realistic compression models, one could determine where and how that cancer will appear in both cranio-caudal and mediolateral oblique mammograms. The model would provide information to radiologists about the 3D shapes and compositions of abnormalities. Additionally, since tumours are rarely seen in a breast screening clinic, the ability to summon up simulations on demand could be a powerful teaching tool as well as proving useful in testing image analysis algorithms and allowing in-service testing of radiologists.

\section{Acknowledgements}

DJG would like to thank the Medical Research Council for a Career Development Fellowship.

\section{References}

Adam, J.A. (1987) "A simplified mathematical model of tumour growth", Math. Biosc. 86, 183-211.

Anderson, A.R.A. and Chaplain, M.A.J. (1998) "Continuous and discrete mathematical models of tumour-induced angiogenesis", Bull. Math. Biol. 60, 857-899.

Anderson, A.R.A., Chaplain, M.A.J., Newman, E.L., Steele, R.J.C. and Thompson, A.M. (2000) "Mathematical modelling of tumour invasion and metastasis", J. Theor. Med. 2, 129-154.

Armitage, P.A., Brady, J.M. (2000) "The effect of t10 correction on contrast-enhanced mri data: errors in concentration". In Proc. ESMRMB 2000

Behrenbruch, C.P., Marias, K., Armitage, P.A., Brady, J.M., Clarke, J.M. and Moore, N. (2000) "The generation of simulated mammograms from contrast-enhanced mri for surgical planning and postoperative assessment", Proceedings of the International Workshop in Digital Mammography (Kluwer Academic Publishers, Dordrecht).

Behrenbruch, C.P., Marias, K., Armitage, P.A., Yam, M., Moore, N., English, R.E. and Brady, J.M. (2000) "Mammography-mri 2d/3d data fusion for breast pathology assessment", Proceedings of MICCAI (Medical Robotics, Imaging and Computer Assisted Intervention) (Springer, New York).

Behrenbruch, C.P., Marias, K., Yam, M., Brady, J.M. and English, R.E. (2000) "The use of magnetic resonance imaging to model breast compression in X-ray mammography for $\mathrm{mr} / \mathrm{X}$-ray data fusion", Proceedings of the International Workshop in Digital Mammography (Kluwer Academic Publisher, Dordrecht).

Benson, D.L., Maini, P. and Sherratt, J.A. (1993) "Diffusion driven instability in an inhomogeneous domain", Bull. Math. Biol. 55, 365-384.

Benson, D.L., Maini, P.K. and Sherratt, J.A. (1998) "Unravelling the turing bifurcation using spatially varying diffusion coefficient", J. Math. Biol. 37, 381-417.

Byrne, H.M. (1997) "The importance of intercellular adhesion in the development of carcinomas", IMA J. Math. Appl. Med. Biol. 14, 305-323.

Byrne, H.M. and Chaplain, M.A.J. (1996a) "Growth of necrotic tumours in the presence and absence of inhibitors", Math. Biosc. 135, $187-216$.

Byrne, H.M. and Chaplain, M.A.J. (1996b) "On the importance of constitutive equations in mechanochemical models of pattern formation", Appl. Math. Lett. 9, 85-90.

Cerneaz, N., D.Phil Thesis (Oxford University, Oxford).

Chaplain, M.A.J. and Stuart, A.M. (1993) "A mathematical model for the diffusion of tumour angiogenesis factor in multicellular spheriods", Math. Biosc. 115, 233-246.

Chaplain, M.A.J., Benson, D.L. and Maini, P.K. (1994) "Nonlinear diffusion of a growth inhibitory factor in multicell spheriods", Math. Biosc. 121, 1-13.

Delingette, H. (1998) "Towards realistic soft-tissue modelling in medical simulation", Proc. IEEE 86, 512-523.

Dillon, R.H. (2001) "Mathematical modeling of vertebrate limb development", In: Maini, P.K. and Othmer, H.G., eds, Mathematical Models for Biological Pattern Formation (Springer, New York), $39-57$.

Fung, Y.C. (1993) Biomechanics: Mechanical Properties of Living Tissues, 2nd ed. (Springer, New York).

Greenspan, H.P. (1972) "Models for the growth of a solid tumor by diffusion", Stud. Appl. Math. 51, 317-340.

Greenspan, H.P. (1974) "On the self-inhibited growth of cell cultures", Growth 38, 81-95.

Heyton, P., Brady, M., Tarassenko, L. and Moore, N. (1997) "Analysis of dynamic mr breast images using a model of contrast enhancement", Med. Image Anal. 1, 3.

Highnam, R. and Brady, M. (1999) Mammographic Image Analysis (Kluwer Academic Publishers, Netherlands).

Highnam, R.P., Brady, M. and Shepstone, B.J. (1996) "A representation for mammographic image processing", Med. Image Anal. 1, 1-19.

Highnam, R.P., Brady, M. and English, R. (1999) "Detecting film-screen shot noise in mammography using a model-based approach", IEEE trans. Med. Imaging 18, 1016-1024.

Hoffman, U., Brix, G., Knopp, M.V., Hess, T. and Lorentz, W.J. (1995) "Pharmacokinetic mapping of the breast: a new method for dynamic mr mammography", Magn. Reson. Med. 33, 506-514.

Jackson, T.L., Senter, P.D. and Murray, J.D. (2000) "Development and validation of a mathematical model to describe anti-cancer prodrug activation by antibody-enzyme conjugates", J. Theor. Med. 2, 93-111.

Kaiser, W.A. (1990) "Dynamic magnetic resonance breast imaging using a double breast coil: an important step towards routine examination of the breast", Frontiers Euro. Radiol 7, 39-68.

Karssemeijer, N. (1993) "Adaptive noise equalisation and recognition of microcalcification clusters in mammograms", Int. J. Pattern Recog. Atrif. Intell. 7, 1357-1376.

Karssemeijer, N., Thijssen, M. and Hendriks, J. (1998) In: van Erning, L., ed, Digital Mammography (Kluwer Academic Publishers, Nijmegen).

Kok-Wiles, S.L., Brady, M. and Highnam, R.P. (1998) "Comparing mammogram paris for the detection of lesions", In: Karssemeijer, N., ed, Fourth International Workshop on Digital Mammography (Kluwer Academic Publishers, Nijmegen), pp 103-108. 
Maini, P.K., Benson, D.L. and Sherratt, J.A. (1992) "Pattern formation in reaction diffusion models with spatially inhomogeneous diffusion coefficients", IMA J. Math. Appl. Med. Biol. 9, 197-213.

Marias, K., Behrenbruch, C.P., Brady, M. and Seifalian, A. (2000) "Nonrigid registration of temporal mammogram pairs via a combination of boundary and internal landmarks", Proceedings of the International Workshop in Digital Mammography (Kluwer Academic Publishers, Dordrecht).

Marias, K., Behrenbruch, C.P., Highnam, R.P., Brady, J.M., Parbhoo, S. and Seifalian, A. (2000) "Quantifying mammographic changes in temporal hrt sequences", Proceedings of MIUA'00 (University College London).

Marušić, M., Bajzer, Ž. and Vuk-Pavlović, S. (1994) "Tumour growth in vivo and as multicellular spheriods compared by mathematical models", Bull. Math. Biol. 56, 617-631.

McCulloch, A.D., Bassingthwaighte, J.D., Hunter, P.J. and Noble, D. (1998) "Computational biology of the heart: from structure to function”, Prog. Biophys. Molec. Biol. 69, 153-155.

Moore, N, Behrenbruch, C., Hardingham, C., Armitage, P., Brady, M. (2001) " $3 \mathrm{~d}$ non-rigid matching of contrast-enhanced mri to assess chemotherapy response in breast cancer". To appear in Proceedings of ECR (European Congress of Radiology).

Murray, J.M. (1997) "The optimal scheduling of two drugs with simple resistance for a problem in cancer chemotherapy", IMA J. Math. Appl. Med. Biol. 14, 283-303.
Netti, P.A., Baxter, L.T., Boucher, Y., Skalak, R. and Jain, R.K. (1997) "Macro- and micro-scopic fluid transport in living tissue: application to solid tumour", AIChe J. 43, 818-834.

Skalak, R., Zargaryan, S., Jain, R.K., Netti, P.A. and Hoger, A. (1996) "Compatibility and the genesis of residual stress by volumetric growth", J. Math. Biol. 34, 889-914.

Stott, E.L., Britton, N.F., Glazier, J.A. and Zajac, M. (1999) "Stochastic simulation of benign avascular tumour growth using the potts model", Math. Comput. Modelling 30(5-6), 183-198.

Sutherland, R.M. (1988) "Cell and environment interactions in tumour microregions: the multispheriod model", Science 240, 177-183.

Swanson, K.R., (1999) "Mathematical modeling of the growth and control of tumours", PhD Thesis (University of Washington).

Swanson, K.R., Alvord, Jr., E.C. and Murray, Jr., J.D. (2000) "A quantitative model for differential motility of gliomas in grey and white matter cell proliferation", Cell Proliferation, 33(5), 317-330.

Tofts, P. and Kermode, A. (1991) "Measurement of the blood-brain barrier and leakage space using dynamic mr imaging 1. Fundamental concepts", Magn. Reson. Med. 17, 347-367.

Ward, J.P. and King, J.R. (1997) "Mathematical modelling of avasculartumour growth", IMA J. Math. Appl. Med. Biol. 14, 39-69.

Yam, M. and Brady, J.M. (1999) "Reconstructing microcalcification clusters in 3-d using a parameterised breast compression model", Proceedings of the Computer Assisted Radiology and Surgery (CARS), (Paris).

Zwiggelaar, R., Parr, T.C. and Taylor, C.J. (1996) Finding Orientated Line Patterns in Digital Mammographic Images (BMVC, Edinburgh). 


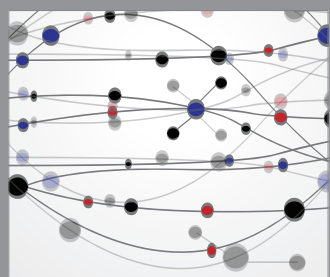

The Scientific World Journal
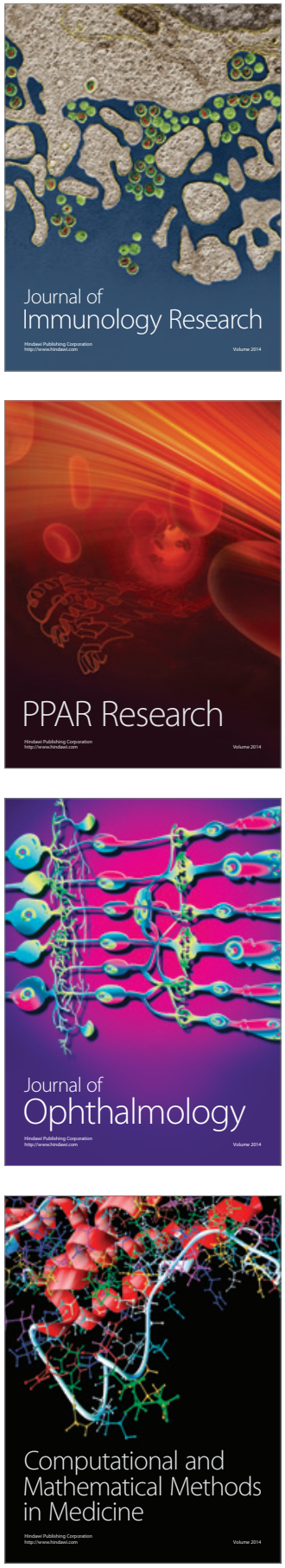

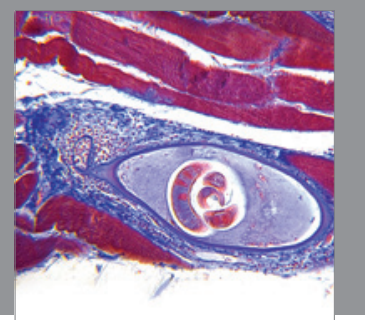

Gastroenterology

Research and Practice
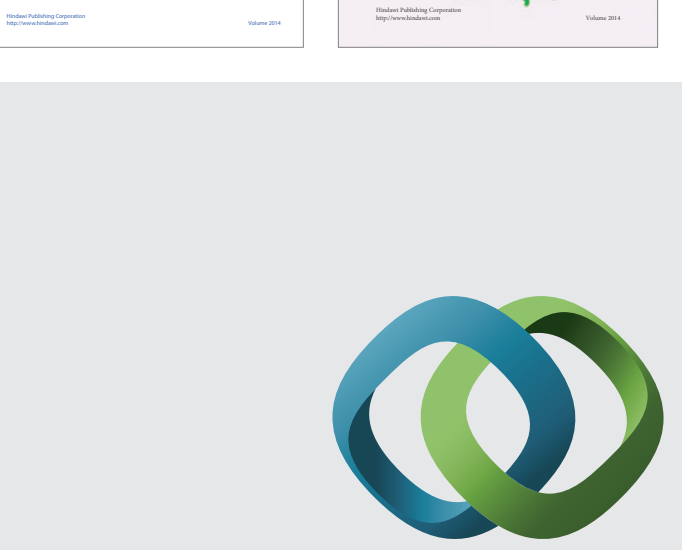

\section{Hindawi}

Submit your manuscripts at

http://www.hindawi.com
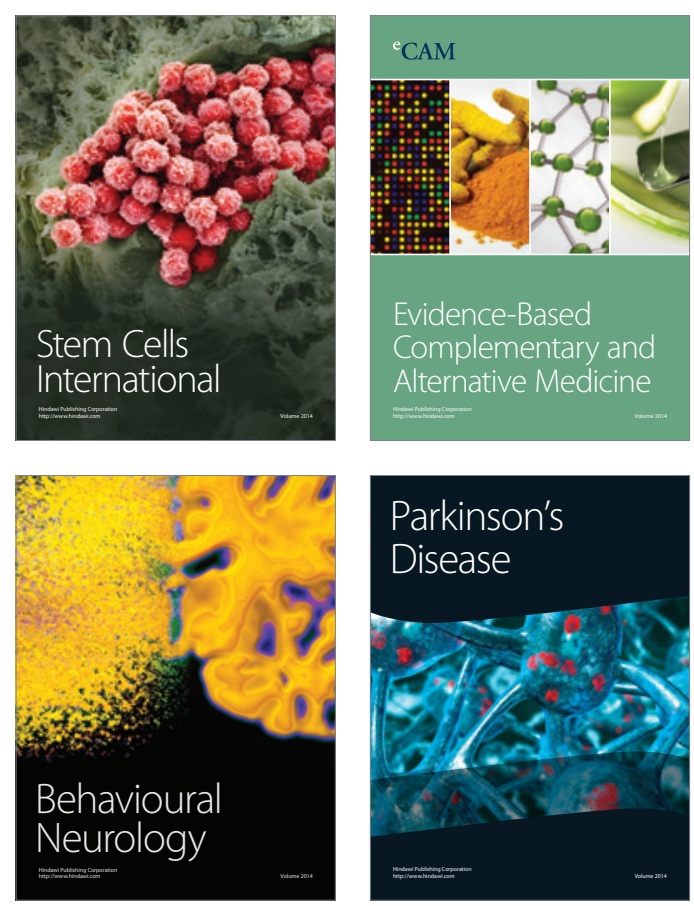

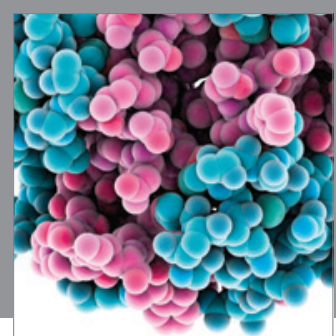

Journal of
Diabetes Research

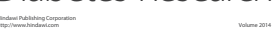

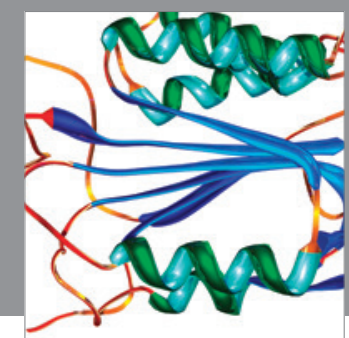

Disease Markers
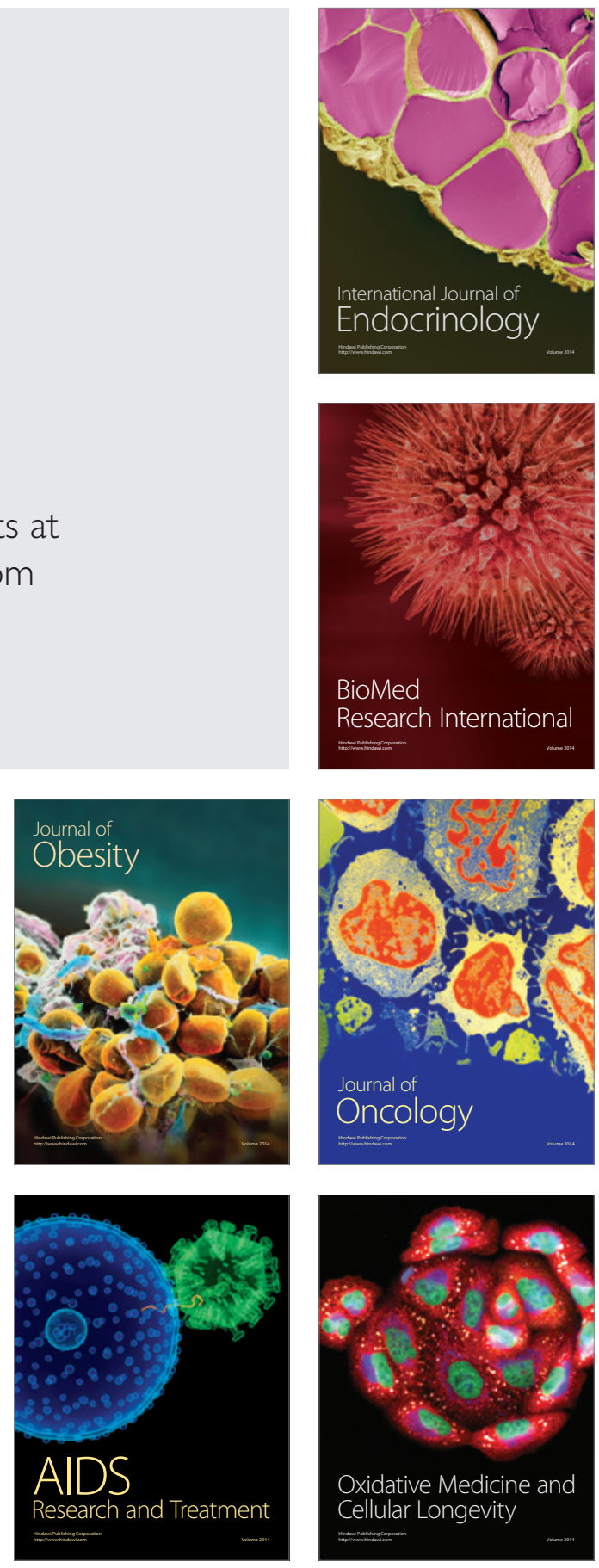